\title{
Good host - bad host: molecular and evolutionary basis for survival, its failure, and virulence factors of the zoonotic nematode Anisakis pegreffii
}

Željka Trumbić ${ }^{1}$, Jerko Hrabar ${ }^{2}$, Nikola Palevich ${ }^{3}$, Vincenzo Carbone ${ }^{3}$, Ivona Mladineo ${ }^{4 *}$

${ }^{1}$ University Department of Marine Studies, University of Split, 21000 Split, Croatia

${ }^{2}$ Laboratory of Aquaculture, Institute of Oceanography \& Fisheries, 21000 Split, Croatia

${ }^{3}$ AgResearch Limited, Grasslands Research Centre, Palmerston North, 4410, New Zealand

${ }^{4}$ Laboratory of Functional Helminthology, Institute of Parasitology, Biology Centre of Czech Academy of Science, 37005 Ceske Budejovice, Czech Republic

\section{corresponding author: ivona.mladineo@paru.cas.cz}


Parasitism is a highly successful life strategy and a driving force in genetic diversity that has evolved many times over. Consequently, parasitic organisms have adopted a rich display of traits associated with survival that guarantees an effective "communication" with the host immunity and a balance with surrounding microbiome. However, gain/loss of hosts along the evolutionary axis represents a complex scenario that as contemporary onlookers, we can observe only after a long time displacement. The zoonotic and monophyletic Anisakidae diverged from its terrestrial sister group Ascarididae 150-250 Ma, although a split from their common ancestral host, a terrestrial amniote, seemingly happened already in Early Carboniferous (360.47 Ma). Faced with the sea-level rise during the Permian-Triassic extinction (215 Ma), anisakids acquired a semiaquatic tetrapod host, and as a result of lateral host-switches in Cenozoic, colonised marine mammals, co-evolving with their "new hosts". Although contemporary anisakids have lost the ability to propagate in terrestrial hosts, they can survive for a limited time in humans. To scrutinize anisakid versatility to infect evolutionary-distant host, we performed transcriptomic profiling of larvae infecting the accidental host (rat) and compared it to that of larvae infecting an evolutionary-familiar, paratenic host (fish). Identified differences and the modeling of handful of shared transcripts, provides the first insights into evolution of larval nematode virulence, warranting further investigation of shared transcript as potential drug therapy targets. Our findings have also revealed some key intrinsic cues that direct larval fate during infection.

Key words: accidental host, anisakiasis, Anisakis spp., drug targets modelling, paratenic host, transcriptomics

\section{Introduction}

Nematodes from genus Anisakis reproduce in marine mammals and propagate embryonated eggs via cetaceans faeces into marine environment, where hatched or in egg-moulted larvae (L1 and L2, or first- and second-stage larvae) use intermediate (copepods and euphausiids) and paratenic (fish, cephalopods) hosts to moult into infective L3 (third-stage larvae), subsequently reaching the gastric chambers of the toothed whales (L4, or fourth-stage larvae, adults) through the trophic chain (see Mattiucci et al, 2018; Mladineo and Hrabar, 2020). In the paratenic hosts, being the most important from the human epidemiology standpoint, larvae perforate gastrointestinal wall and spiralise on visceral surface of abdominal organs in a dormant stage of paratenesis (Beaver, 1969). Therefore, the level of tissues damage in paratenic hosts is usually limited and reversible, although persistent infections and an elevated number of dormant larvae might interfere with its fitness (Levsen et al., 2018). More importantly, infective L3 can also migrate intra vitam or post mortem into paratenic hosts' musculature (Cipriani et al. 2016).

Humans are considered only accidental hosts that contract L3 through consumption of inadequately prepared seafood, therefore the associated diseases have mainly been recognised in countries with larger per capita fish consumption (Pozio, 2013). Described for the first time in 1960 in the Netherlands (Van Thiel, 1960), human anisakiasis is a disease caused by infective larvae of genus Anisakis spp. (Anisakidae, Nematoda), occurring as gastric, intestinal, ectoptic or gastro-allergic form (Audicana and Kennedy, 2008), or eventually as an asymptomatic form within Anisakis-seropositive population (Moneo et al., 2017). Although it shows an ambiguous epidemiological status worldwide (Bao et al., 2019), anisakiasis listed as fifth in the European risk ranking, and the second of 24 foodborne parasitoses with the highest "increasing illness potential" (Bouwknegt et al., 2018). Nonetheless, it is still considered underreported in Europe, 
showing a large bias between clinical reported cases, i.e. 236 between 2000-2016 (Serrano-Moliner et al., 2018), and the predicted annual number deriving from a quantitative risk assessment model, i.e. between 7,700-8,320 only in Spain (Bao et al., 2017).

The damage afflicted in humans as accidental hosts during larval migration has been extensively reviewed (Audicana and Kennedy, 2008; Hochberg and Hamer, 2010), and rodent models mimicking humans as host-type have been used to characterise Anisakis sp. pathogenicity and virulence (Romero et al., 2013; Zuloaga et al., 2013; Lee et al., 2017; Bušelić et al., 2018; Corcuera et al., 2018; Hrabar et al., 2019) (excluding sensitization/ allergy studies). In experimentally Anisakis-infected rats, the early response consists of a strong immune reaction with marked induction of specific proinflammatory cytokines and alarmins or damage associated molecular patterns (DAMPS; calprotectins S100A8 and S100A9), regulated through expression of leukocytes-silencing miRNA (miRNA-451 and miRNA-223) (Bušelić et al., 2018; Hrabar et al., 2019). Conversely, Anisakis experimentally infecting fish induce no significant regulation of cytokines (IL-1 $\beta$, IL-4/IL-13, IL-6, IL-8, IL-10, IL-22, TNF $\square$ and TGF $\beta$ ) and downregulation of IgM and CD8 (cytotoxic $\mathrm{T}$ cells), suggesting that larval excretory/ secretory products (ESPs) contribute to the immune silencing in this particular host (Haarder et al., 2013). The only significant early upregulation of serum amyloid A (SAA) in liver, an acute-phase protein, evidences for a systematic proinflammatory reaction that subsides by time. This may be the cause of spontaneous resolving of Anisakis infections in fish under the experimental conditions, where only few larvae successfully fulfil their role within their natural paratenic host (Quiazon et al., 2011; Haarder et al., 2013; Marino et al., 2013). However, this greatly contradicts the epidemiological omnipresence of anisakids in fish in nature (Levsen et al., 2018).

We hypothesize that each particular host-type, i.e. paratenic and accidental, represents a distinct ecological niche for the parasite that consequently prompts the infective stage to incur dramatically different strategies, eventually leading to a different degree of propagation success within the host. Such "strategies", translated through the parasite's transcriptomics signatures, could be very useful to infer factors of virulence, as well as array of adaptational traits necessary for parasite survival, development and interaction with the host that will allow the parasite to thrive. The features of both the virulence [herein defined as a reduction in host fitness caused by infection (Read, 1994), not to be interchangeably used with pathogenicity, which refers to the ability of an organism to cause disease] and adaptations, depicted as perturbed gene groups and pathways, could be in future used for targeted drug therapy.

Therefore, we aimed to evaluate the early $(<32 \mathrm{~h})$ transcriptomic response of Anisakis pegreffii migrating and non-migrating L3 during experimental infection of an evolutionary-familiar host (i.e. fish, paratenic host) and evolutionary-distant host (i.e. rat, accidental host and model for human infection). We further assumed that the transcripts significantly changed between migrating and non-migrating L3 and common denominators for both hosts would indicate genes essential for successful larval propagation. Finally, to gain insight into the substrate specificities of the genes deemed important for Anisakis virulence, we modelled the tertiary structures using the full-length amino acid sequences of a set of differentially expressed genes that are common in both host-types and inferred the size of their homologous families within available helminth genomes.

\section{Materials and Methods}

\section{Animal Ethics}

All animal care and use protocols were conducted in accordance with ethical standards for animal protection. Experimental infections on rats were performed at the Animal Facility, University of Split (permit number HR-POK-19), approved by the Veterinary and Food Safety Authority, Ministry of Agriculture of the Republic of Croatia (permit number EP 18-2/2016) and Ethics Committee of the University of Split, School of Medicine (permit number 003-08/18-03/0001). 
Rats were raised and housed in pairs, in plastic cages with sawdust and corn bedding, food and water ad libitum, temperature $22 \pm 1{ }^{\circ} \mathrm{C}$, with a $12 \mathrm{~h}$ light/dark cycle (Bušelić et al, 2018). The experimental infections of the European sea bass (Dicentrarchus labrax) were performed at the experimental hatchery facilities of the Institute of Oceanography and Fisheries. Fish $(n=32)$ were transported from a nearby farm and transferred to a single concrete flow-through tank $\left(12 \mathrm{~m}^{3}\right)$ were they were fed commercial dry formulated diet and kept under natural photoperiod. Water parameters (salinity, temperature and dissolved oxygen) were measured daily by OxyGard H01PST probe. The fish were then distributed into additional four tanks for 15 days acclimatization prior to experimental infections, so that each tank contained seven fish, and the fifth accommodated four fish that served as a negative control.

\section{Experimental Infections}

Anisakis spp. larvae were collected from natural sources. Blue whiting Micromesistius poutassou were freshly caught and immediately iced by commercial fishermen in the Adriatic Sea and delivered to experimental facilities on the morning of the experiment. Actively moving larvae were carefully removed with forceps from fish viscera and washed in a physiological saline solution. Integrity of their cuticle was checked under a stereomicroscope (Olympus BX 40) and larvae were kept at $4{ }^{\circ} \mathrm{C}$ until experimental manipulation, when they were placed in gastric probes. At this point, a sample of pre-infection larvae was preserved in Tri Reagent (Ambion Inc., Invitrogen, Carlsbad, CA, USA) at $-80{ }^{\circ} \mathrm{C}$ for RNA extractions.

The duration of experimental infections with the two hosts was determined in preliminary experiments. For the detailed description of preliminary findings and the setup of the final experiment on rats, the reader is referred to Bušelić et al. (2018). Briefly, rats were separated in individual cages $24 \mathrm{~h}$ prior to the experiment and deprived of food. Thirty-five male Sprague-Dawley rats were used for the final experiment (average weight $207 \pm 20.1 \mathrm{~g}$ ), split into five groups each sacrificed at $6,10,18,24$ and $32 \mathrm{~h}$ post-infection. Within each group, five rats were intubated with 10 Anisakis pegreffii larvae each and two were intubated with 1.5 $\mathrm{mL}$ of physiological saline solution serving as external controls. For the intubations, rats were anesthetized using a mixture of 50-100 mg/kg of Ketaminol (Richter Pharma AG, Wels, Austria) and 5-10 mg/kg of Xylapan (Vetoquinol UK Ltd, Buckingham, UK) by intraperitoneal injection. At specific post-infection time-points, animals were administered an overdose of Ketaminol (Richter Pharma AG, Wels, Austria; > $150 \mathrm{mg} / \mathrm{kg}$ ) and decapitated to confirm death. Following dissection and gross pathological examination, damaged host tissue and recovered A. pegreffii larvae were collected and stored in Tri Reagent (Ambion Inc., Invitrogen, Carlsbad, CA, USA) at $-80^{\circ} \mathrm{C}$. Prior to conservation, larvae were washed in physiological saline solution to remove all visible traces of host tissue.

Similarly, European sea bass were deprived of food $24 \mathrm{~h}$ prior to the experimental intubation with $A$. pegreffii larvae. In total, 32 male sea bass (average weight $92.9 \pm 22.2 \mathrm{~g}$, average length $20.8 \pm 1.8 \mathrm{~cm}$ ) were used for this experiment, 28 intubated with L3 larvae and four used as external controls intubated with $2 \mathrm{~mL}$ of physiological saline solution. Fish were anesthetized by submersion bath into tricaine methanesulfonate (MS222, Sigma Aldrich, St. Louis, Missouri, United States) solution in seawater (15-30 mg/L) and each intubated with $20 \mathrm{~A}$. pegreffii larvae or physiological saline solution. The duration of the experiment was limited to $12 \mathrm{~h}$ as we observed a high rate of larvae clearance during a preliminary experiment (data not shown) via passage through digestive tract or regurgitation. Sea bass were euthanized at 3, 6, 9 and $12 \mathrm{~h}$ post-infection (7 fish per time-point including controls) by an overdose of MS222. Animals were inspected as previously described for the rat experiment and samples were stored for further analyses.

\section{Extraction of Nucleic Acids}

Total RNA was extracted from total sampled larvae for transcriptomic analyses and DNA was subsequently extracted from reaction leftovers in order to identify Anisakis species at molecular level. By doing so we managed to use molecular information from an entire larva for transcriptomics and avoid possible losses due to tissue fragmentation that would be required for separate DNA preparations. Total RNA was extracted using Tri Reagent (Ambion Inc., Invitrogen, Carlsbad, CA, USA) as per manufacturer's 
instructions and dissolved in 20-40 $\mu$ l of RNase/DNase free water (Merck Millipore, Billerica, MA, USA). Quantity, purity and integrity of RNA preparations were assessed by spectrophotometry and $1 \%$ agarose gel electrophoresis, respectively. DNA was extracted from Tri Reagent discarded after phase separation for each individual RNA extraction as described in the manual. Molecular identification of larvae was performed using PCR-RFLP method described by D'Amelio et al. (2000). Restriction pattern of rDNA characteristic of Anisakis simplex (sensu stricto) $\times$ A. pegreffii putative hybrid was observed for a single larva and all other were confirmed as A. pegreffii.

\section{Library Preparation for Illumina Sequencing}

Samples were sequenced at the Laboratory for Advanced Genomics, Division of Molecular Medicine at the Ruđer Bošković Institute, Zagreb, that also performed cDNA library preparation. Quality and quantity of RNA extractions were checked using a 2100 BioAnalyzer (Agilent Technologies, Santa Clara, CA, USA) and Qubit 3.0 (Thermo Fisher Scientific, Waltham, MA, USA). Based on the sample quality and the experimental outcome, 16 pools of several biological replicates were created for larvae used in rat infections, 8 for migrating larvae, 6 for non-migrating and one pool of naïve pre-infection larvae. We preserved the information about the tissue of larvae recovery within the host (Supplementary Table 1). Larvae sampled from sea bass each constituted individual samples with 4 libraries for non-migrating, postmigrating and spiralized larvae and 4 for larvae collected prior to the start of the experiment (Supplementary Table 1). cDNA libraries were prepared according to TruSeq Stranded mRNA kit (Illumina, San Diego, CA, USA). The paired-end sequencing (75 bp from each end) was performed on the Illumina NextSeq 500 platform (Illumina, San Diego, CA, USA) over four lanes.

\section{RNA-Seq Raw Reads Pre-processing}

FASTQC v0.11.8 (Andrews, 2018) was used to assess read quality and tailor all subsequent read cleaning steps. Trimmomatic v0.39 (Bolger et al, 2014) was used to remove Illumina adapter sequences, cut first 13 bases from the start of a read due to per base sequence content bias, clip the reads using a sliding window of 4 bases with quality threshold of 20 and remove reads shorter than 30 bases, in that order. The proportion of ribosomal RNA (rRNA) reads in each library was assessed and removed using SortMeRNA v2.1 by comparison against rRNA databases included in the software package (SILVA and RFAM) (Kopylova et al, 2012). Finally, reads were screened for host contamination by mapping against respective host genomes, Rattus norvegicus (v6), $\quad$ Ensembl release 98 (https://www.ensembl.org/Rattus_norvegicus/Info/Index) and Dicentrarchus labrax (dicLab v1.0c available from http://seabass.mpipz.mpg.de/cgi-bin/hgGateway) using STAR v2.7.1a (Dobin et al, 2013) in 2-pass mapping mode. Reads that failed to map to respective host genomes were used for downstream analyses.

\section{Transcriptome Assembly and Functional Annotation}

Paired-end reads passing quality control were concatenated across all samples into a single set of inputs as recommended by Haas et al (2013) and used to reconstruct a reference transcriptome using Trinity v2.8.6. (Grabherr et al, 2011) with default parameters (kmer size 25, minimum contig length 200 nucleotides, strand-specific read orientation set to RF). In order to assess the quality of the initial assembly, we calculated assembly N50 (by TrinityStats.pl script from Trinity), overall read alignment rate using Bowtie2 v2.3.5.1 (Langmead et al, 2018) and examined the number of transcripts that appear to be nearly full-length by comparing assembled transcripts against UniProtKB/Swiss-Prot database (The UniProt Consortium, 2019). For this purpose, blastx algorithm (Altschul et al, 1990) was used as implemented in BLAST+ v2.10.0 (Camacho et al, 2009), with an expectation value ( $e$-value) cut-off of $10^{-20}$. Conserved ortholog content of the reference assembly was assessed using BUSCO v3.0.2 (Waterhouse et al, 2017) in transcriptome mode against metazoa_odb9 (creation date: 2017-02-13). To reduce transcriptome redundancy and concentrate on potential coding regions, CD-HIT-EST v4.8.1. (Li and Godzik, 2006) was first used to cluster sequences on a nucleotide level using similarity threshold of 0.99. Open reading frames (ORFs) were 
predicted on this set using TransDecoder v5.5.0 (Haas et al, 2013) with a minimum protein length of 80 amino acids. Predicted ORFs were searched against Uniprot/SwissProt database using blastp (e-value cut-off of $10^{-5}$ ), while HMMER v3.2.1. (Eddy, 2011) was used to compare peptides against PFAM database v31.0. (El-Gebali et al, 2019) to identify common protein domains. Single best open reading frame (ORF) per transcript was retained using TransDecoder --single_best_only pipeline prioritizing homology hits over ORF length. Redundancy was further reduced in the remaining transcript set by clustering highly similar amino acid sequences with CD-HIT v4.8.1 ( $\mathrm{Li}$ and Godzik, 2006), using an identity threshold of 1.00. Coding sequences were annotated using blastp (e-value cut-off of $10^{-5}$ ) against The NCBI Reference Sequence (RefSeq) collection of non-redundant proteins (release96, accessed $15^{\text {th }}$ Dec 2019 at https://www.ncbi.nlm.nih.gov/refSeq), as well as against publicly available proteomes of model and closely related nematodes respectively: Caenorhabditis elegans (WBcel235, Ensemble release 98), A. simplex (A_simplex_0011_upd) and Ascaris lumbricoides (A_lumbricoides_Ecuador_v1_5_4), available from WormBase ParaSite (https://parasite.wormbase.org). Trinotate v3.1.1 (Bryant et al, 2017) annotation suite was used to retrieve GO (Gene Ontology), KEGG (Kyoto Encyclopedia of Genes and Genomes) and eggNOG (evolutionary genealogy of genes: Non-supervised Orthologous Groups) annotations from blast results and collect all functional information into a SQLite database used to filter and sort data as needed.

\section{Differential expression analysis}

Paired-end reads of each sample were mapped to the reference transcriptome as strand specific using Bowtie2 v2.3.5.1 (Langmead et al, 2018) and abundances estimated by RSEM v1.3.1 (Li and Dewey, 2011) with default parameters. Gene level estimated counts were imported into R v3.6.3 (R Core Team, 2020) through tximport package (Soneson et al, 2015). Differential analysis of gene expression was performed using DESeq2 package (Love et al, 2014) for Biocondutor v3.10 (Huber et al, 2015). Prior to statistical testing, low count transcripts (with less than four reads summed across at least four samples) were prefiltered and removed from the dataset and exploratory data analysis was performed using principal component analyses (PCA) to ensure correct grouping of the samples according to phenotype. Read counts were modelled as following a negative binomial distribution, and a generalized linear model was fitted for each gene with multi-factor design that included host, state (migrating vs non-migrating) and the interaction term as fixed effects. The results were generated for three contrasts in total using the Wald test: migrating vs. non-migrating larvae in rat, migrating vs. non-migrating larvae in sea bass and the difference between the two, i.e. the interaction showing if the regulation was different when considering migrating vs. nonmigrating A. pegreffii larvae in two distinct hosts. Pre-infection larvae collected directly from fish prior to the experiment were not included in the statistical testing as this was not the primary experimental question. Differentially expressed genes (DEGs) were identified at Benjamini-Hochberg false discovery rate (FDR) < 0.05 without fold change cut-off. Expression profiles for DEGs were explored and visualized using a venn diagram generated by VennDiagram (Chen, 2018) and ggplot2 packages (Wickham, 2016) for R. Five target DEG sequences; ATP-binding cassette transporter $a b c b 9$, UDP-glucuronosyltransferase ugt, aspartic protease asp6, leukotriene A4 hydrolase lkha4 and cytosolic non-specific dipeptidase cndp2, common for both hosts and therefore considered as virulence factors, were selected for downstream analyse of gene family evolution and protein modelling.

In order to gain whole-systems understanding of the data, enrichment of GO terms and KEGG pathways via KEGG Orthologue (KO) identifiers within sets of DEGs was calculated using goseq package, taking gene length bias into account (Young et al, 2010). Enrichment within specific DEG group was tested against all expressed genes (after removal of low count features) as a background. Terms/pathways were identified as significantly enriched/unenriched at Benjamini-Hochberg false discovery rate (FDR) $<0.05$. For this purpose, KAAS (KEGG Automatic Annotation Server) was used to obtain more extensive KO mapping for Anisakis putative peptides using SBH method (Moriya et al, 2007). Following enrichment analyses that did not produce conclusive results, the frequency of all DEGs per GO terms and KEGG 
metabolic and signaling pathways was calculated and inspected next to mean log (base 2)-fold changes ( $\log 2 \mathrm{FC})$ per term/pathway.

\section{Gene Family Evolution}

The five target DEG protein sequences common for both hosts were searched against the Wormbase Parasite (Howe et al., 2017) protein BLAST database using the BLASTP version 2.9.0+ (Camacho et al., 2009) search with default settings. Proteomes containing hits with over 70\% identity, scores at least $50 \%$ of the $A$. pegreffii match and hit sequence lengths at least $75 \%$ of the query sequence lengths were downloaded from WormBase. We gathered predicted proteomes of selected Nematoda representing Clades III, V, IV, C, I, Platyhelminthes belonging to Clades Monogenea, Trematoda, Cestoda, Rhabditophora, with Homo sapiens, Mus musculus and Danio rerio as outgroups. To determine homologs of the five target A. pegreffii DEGs, we identified single-copy orthologous groups (OGs) in the five proteomes using OrthoFinder v 2.5.2 (Emms et al., 2015), with cluster selection based on at least $75 \%$ species present with a single protein in each cluster. The sequences of the proteins in the OGs containing the five target proteins were extracted and searched against the "nr" database using the BLASTP function of diamond version 2.0.6 (Buchfink et al., 2015) with default settings. InterProScan version 5.26-65.0 (Jones et al., 2014) was used to annotate protein domains and GO terms in the extracted proteins. The diamond BLASTP and InterProScan results were merged using OmicsBox version 1.4.11 (BioBam Bioinformatics, 2019). The extracted proteins were subjected to phylogenetic analysis with 1,000 bootstrap replicates for full-coverage data performed using MAFFT (Katoh and Standley, 2014). Also, individual maximum likelihood (ML) inferences for each resulting trimmed gene-cluster were generated to infer the species tree under a multiple-species coalescent model. An evolutionary model was selected automatically for each cluster and visualized in Geneious Prime v.2019.1.3 (Kearse et al., 2012).

\section{Protein modelling and structural analysis}

The Position-Specific Iterative Basic Local Alignment Search Tool (PSI-BLAST) (Altschul et al., 1997) was used to compare the protein sequences associated with the DEGs of interest with the corresponding deposited structures in the Protein Data Bank (PDB). Structural models of the set of DEGs that are common in both host-types were constructed by submitting the obtained amino acid sequences to the I-TASSER server (Yang et al., 2015). The modelled DEGs include: cytosolic non-specific dipeptidase (CNDP2, E.C. 3.4.13.18), leukotriene A-4 hydrolase (LKHA4, E.C. 3.3.2.6), aspartic protease 6 (ASP6, E.C. 3.4.23.3), ATP-binding cassette sub-family B member 9 (ABCB9, E.C. 7.6.2.2) and UDPglucuronosyltransferase (UGT3, E.C. 2.4.1.17). To assess the quality of the model and residue geometry, TM-scores (a metric for measuring the similarity of two protein structures and fold similarity) (Zhang and Skolnick, 2004) and C-scores (confidence score of the predicted model) all fell within predicted ranges depicting high confidence in our structures. The best model obtained for each DEG was then further validated using Procheck (Laskowski et al., 1996) and ProSA-web (Wiederstein and Sippl, 2007). The active site residues were deduced and visualized using PyMOL Molecular Graphics System v2.0 (Schrodinger, 2010) where the model was superimposed with their parent structures co-crystallized with substrate or inhibitor.

\section{Data availability}

Raw sequence reads were submitted to NCBI Sequence Read Archive (SRA) and are available under BioProject accession PRJNA475982 (runs SRR13870203 - SRR13870233). 


\section{Results}

\section{Description of the experimental outcome}

The outcome of experimental infections of Sprague-Dawley rats with A. pegreffii L3 larvae have been previously described in Bušelić et al (2018) and Hrabar et al (2019) detailing host tissue damage and response through histopathology, TEM analyses, immunofluorescence screening, miRNA and transcriptomic profiling. Here we focus on the infecting larvae display and briefly recap some of those results as to facilitate the comparison with the experimental outcome in sea bass. In rats, most of the larvae were found passing through the digestive tract (51\%), designated as i) non-migrating, as they did not engage in host tissues penetration (Table 1); ii) actively penetrating (migrating through) host gastric and intestinal wall or abdominal wall muscles $(37.8 \%)$. The latter caused mild to severe haemorrhages visible upon gross pathological examination, oedema, inflammatory infiltrate, compression and necrosis. A few larvae were found dwelling in the abdominal cavity presumably after active penetration of host gastrointestinal tract, designated as iii) post-migratory $(6.1 \%)$, or iv) in the early stages of spiralization, i.e. settling on the serosa of internal organs and peritoneum $(5.1 \%)$ (Table 1$)$.

Larvae showed no synchronized behavior in respect to specific time post-infection and were found at different migratory stages at all sampling points, although the incidence of non-migratory larvae has decreased with increasing time post-infection and the incidence of all migratory phenotypes has accordingly increased (Table 1). However, larvae clearance also intensified with the passage of time as median recovery rate was $90 \%$ at $6 \mathrm{~h}$ post-infection and only $25 \%$ at $32 \mathrm{~h}$ post-infection (Table $\mathbf{1}$ ).

In contrast to the outcome observed with the accidental host, experimental infections with sea bass, a paratenic host, demonstrated much larger clearance rate with median recovery of only $12.5 \%$ at $3 \mathrm{~h}$ postinfection and 0 at $9 \mathrm{~h}$ (Table 1). Large individual variability was observed for sea bass at each sampling point, with some fish clearing all intubated larvae, hence the time duration of the experiment was reduced and number of intubated larvae per animal increased in respect to rats. The vast majority of larvae were found passing through the digestive tract $(67.6 \%)$, followed by larvae dwelling in the abdominal cavity or initiating spiralization on the surfaces of liver, stomach, gall bladder or visceral fat (15.5\% each) and a single larva (1.4\%) was found during active migration penetrating the wall of the small intestine (Table 1). In the latter case, no gross pathological signs of the process were observed, except for a minor perforation. This larva was sampled for a purpose different from RNA-seq (Hrabar et al 2019), therefore was not included in this study. All larvae were collected for further analyses, however due to rapid time-course of infection in sea bass and inconspicuous penetration of the larvae, we were not able to collect host tissue samples that would allow us to match the level of investigation we were able to conduct for rat.

The experimental outcomes guided our choice of samples for transcriptome analyses. As most larvae were found actively penetrating rat abdominal muscles, sample pools were created grouping migrating larvae from different post-infection points in respect to those non-migrating, in order to gain robust estimates of $A$. pegreffii larvae gene expression signatures important for the process of initiating host penetration. In sea bass, each library constituted individual larva in different stages of migration (nonmigrating, post-migrating, spiralized) as we were not able to construct meaningful pools with the samples obtained.

\section{Anisakis pegreffii reference transcriptome}

In total, $31 \mathrm{~A}$. pegreffii samples were sequenced producing on average 28.8 million read pairs per library. On average, $84 \%$ of read pairs per sample survived quality filtering steps and a total of 736,718,931 read pairs was used as input for transcriptome reconstruction using Trinity v2.8.6. (Grabherr et al, 2011). Initial de novo transcriptome consisted of 141,685 transcripts with a total of 189,360,713 assembled bases, median contig length of 596 and with N50 value of 2,774 (Table 2). Vast majority of input reads was represented by the assembly as their overall alignment rate using Bowtie2 v2.3.5.1 (Langmead et al, 2018) 
was $99 \%$. A quarter of assembled transcripts $(35,704)$ produced a blastx hit with UniProtKB/Swiss-Prot database. There were 4,016 proteins represented by nearly full-length transcripts, having $>80 \%$ alignment coverage. The most targeted organism among blast results was $C$. elegans (CAEEL) with 12501 hits. An assessment of conserved ortholog content according to BUSCO recovered $91.8 \%$ of near-universal complete orthologs from the 978 in the Metazoa database with a few fragmented and missing (C: $91.8 \%$ [S:27.8\%, D:64.0\%], F:1.0\%, M:7.2\%, n:978).

After redundancy reduction by clustering of similar sequences and cleaning, final transcriptome was filtered for transcripts with a detectible coding sequence using TransDecoder v5.5.0 (Haas et al, 2013). It was reduced to 36,201 transcripts with predicted ORFs (Table 2) that preserved most of the conserved orthologue content of the initial assembly (BUSCO report: C: $90.7 \%$ [S:58.5\%, D:32.2\%], F:1.0\%, M:8.3\%, $\mathrm{n}: 978)$. Between 35 and $78 \%$ of these sequences were annotated with different public protein databases (Table 2), with most hits produced with conspecific A. simplex proteome, as expected. Read pairs from each sample were back-mapped to this final transcriptome for differential expression analyses where average overall alignment rate of $88 \%$ per sample was achieved. The final reference transcriptome with nucleotide and amino acid sequences as well as functional annotation is available as Supplementary Table 2.

\section{What is specific about A. pegreffii larvae infecting a paratenic and an accidental host?}

Overall pattern of gene expression observed in collected A. pegreffii L3 larvae during experimental infections of a paratenic, a sea bass, and an accidental host, a rat, is outlined using principal component analyses (PCA) in Figure 1. Consistent with our observations during the experiment, there was high variability between samples mirroring unsynchronized behavior of larvae during the experiment that demonstrated no predilection for a site of infection or temporal dynamics of host invasion. The largest source of variance in the data was the host, as samples primarily grouped whether originating from a homeothermic or poikilothermic host, i.e. rat or sea bass/blue whiting, respectively. Of the two, the strength of the response of larvae that managed to infiltrate host abdominal cavity, was much greater in rat than in sea bass (Figure 1). A single sample of migrating larvae found penetrating rat intestine presented an outlier going against general trend of variance observed for experimental groups and was removed prior to differential expression analyses.

Primary experimental question was to unearth general signatures of gene expression paramount for the process of host infection, i.e. those that would be in common to larvae infecting a rat and a sea bass. In turn, delineation of genetic activity that is different between these two hosts might elucidate evolutionary adaptations of $A$. pegreffii important for its survival and propagation through the trophic chain or what is missing when it encounters an unexpected host such as a rat, or human. The statistical design for differential gene expression analyses was constructed with these questions in mind. All samples derived from larvae that passed host barriers were considered migrating and compared against all those that failed to do so within the same host. Pre-infection larvae collected prior to the start of the experiment were not included in the analyses and are shown for exploratory purposes only.

After removal of low count genes, 1,937 were found differentially expressed at FDR $<0.05$ in migrating vs. non-migrating larvae in rat (1,096 up and 841 down), 484 in sea bass (328 up and 156 down) and 509 showing evidence of interaction between the main effect and the host, i.e. they were differently regulated in the two hosts. Ten DEGs were at the intersection between all three groups (Figure 2) suggesting their importance as contrasting factors in A. pegreffii infection of an accidental and a paratenic host. Largest difference in $\log 2 \mathrm{FC}$ between migrating larvae in rat and in sea bass was observed for a putative cuticle collagen (CO155) and glucose-6-phosphate exchanger (G6PT1) that were both upregulated in rat and downregulated in sea bass. The reverse was true for $\mathrm{NADH}$-dependent flavin oxidoreductase (NADA) (Table 3). Another 65 transcripts were found differentially expressed in migrating larvae in rat and in sea bass showing consistent regulation in both hosts and these are deemed paramount for the process of host invasion in A. pegreffii. Of these, 35 that demonstrated at least two-fold change in gene expression in at least one of the hosts are depicted in Table 3. There is a group of putative collagen transcripts upregulated 
in migrating larvae in both hosts, however more strongly so in larvae infacting a rat than a sea bass. Several catalysts and transporters also feature the list showing moderate and congruent upregulation in both hosts, such as cytosolic non-specific dipeptidase (CNDP2), leukotriene A-4 hydrolase (LKHA4), aspartic protease 6 (ASP6), ATP-binding cassette sub-family B member 9 (ABCB9), UDP-glucuronosyltransferase (UGT3), some of which were chosen as potential drug targets. Unfortunately, we were not able to annotate most strongly upregulated DEGs in this list.

Enrichment analyses of GO terms and KEGG pathways resulted in only few significant terms/pathways (FDR < 0.05) for rat DEGs and almost none for other groups (Supplementary Table 5 and 6). Over-representation of GO terms: structural constituent of ribosome, structural constituent of cuticle, translation, ATP synthesis coupled proton transport, was found in rat DEGs, as well as enrichment of functions associated with KEGG pathways Ribosome and Oxidative phosphorylation. Since enrichment analyses did not provide conclusive results, to observe DEGs from other groups at a more systemic level we investigated DEG frequency distribution for each GO term and KEGG signaling and metabolic pathway alongside average Log2FoldChange (Figure 3). Consistent with the results of enrichment analyses, most upregulated DEGs in rat were counted in KEGG pathways Ribosome, Biosynthesis of secondary metabolites, Oxidative phosphorylation, Microbial metabolism in diverse environments and Carbon metabolism, that also feature as most numerous for sea bass, except for the Ribosome. Two other most represented in sea bass are Lysosome and Autophagy - animal and, although not at the top of the list, there are several DEGs in sea bass associated with drug and xenobiotic metabolism. Most down-regulated DEGs were counted in Endocytosis and Ubiquitin mediated proteolysis in rat, and MAPK signaling pathway and Aminoacyl-tRNA biosynthesis in sea bass. Functional characterization of DEGs based on their frequency in GO categories supports KEGG findings (Supplementary Figure 1). Two interesting biological processes grouping several DEGs from rat and sea bass in GO frequency distribution are collagen and cuticulin-based cuticle development and molting cycle showing relatively high average log2FoldChange $(>2)$.

Gene-level raw counts used for expression analyses are available in Supplementary Table 3 and overall results of differential expression analyses for A. pegreffii larvae from both hosts are presented in Supplementary Table 4 were they can be easily filtered to obtain specific DEG groups according to the venn diagram in Figure 2.

\section{Orthology and evolution of significant DEGs}

We studied the evolutionary significance of ABCB9, UGT3, ASP6, LKHA4 and CNDP2 proteins in eukaryotes. In particular, we searched the complete genomes or transcriptomes and acquired genome-wide coding sequences from 28 species representing clades I-V of nematoda, platyhelminths and free-living flatworms (monogenea, digenea, cestoda), as well as human, mice, zebrafish as outgroups. For this analysis, we used orthologous groups (OGs) of genes identified based on the five selected and significantly differentially expressed genes of interest for $A$. pegreffii and we modeled gene gain and loss for the five orthologues. Comparative analysis within ABCB9 (OG0000006), UGT3 (OG0001081), ASP6 (OG0000081), LKHA4 (OG0001209) and CNDP2 (OG0000954) revealed numerous highly conserved enzymes present across all nematode clades, but with markedly different orthology profiles (Figure 4). Our phylogenetic analyses indicate that ABCB9s are a broad and ancient eukaryotic gene family, with the only loss reported for Macrostomum lignano (Rhabditophora). The UGT3s appear to have been lost independently within eukaryotes, especially in several nematoda (Clades IV and C), Trematoda and Cestoda lineages. In contrast, ASP6s, LKHA4s and CNDP2s appear to be broadly conserved across all species compared in our study, with the exceptions of Fasciola hepatica (ASP6), both Clade I nematodes (LKHA4), and Brugia malayi (CNDP2). Our preliminary orthology analysis of the ASP6 and ABCB9 transcripts of $A$. pegreffii produced a substantial number of overall homologous sequences ( $n=696$ for ABCB9, $n=296$ for ASP6) due to the large and diverse nature of single and multi-domain architectures of eukaryotic ABC transporter/ATP-binding proteins and aspartyl proteases (Supplementary Table 7). To address this, we subdivided clusters of homologous sequences into orthologous groups that matched our A. pegreffii 
transcript annotations and the Enzyme Commission number (E.C.) profiles (Supplementary Tables 2 and 7). In particular, Clade V group nematodes, exhibited massive expansions of ASP6s and UGTs, with tens of homologs that broadly cluster in the phylogeny into two different groups.

\section{D structure of selected virulence factors and their potential drug-targeting}

The five DEGs of interest were modeled using the I-TASSER server to produce tertiary structures of the enzymes (Figure 5). The best of these structures included the ATP-binding cassette transporter ABCB9 (E.C. 7.6.2.2) and the UDP-glucuronosyltransferase UGT3 (E.C. 2.4.1.17; Fig. 5A and C) with C-scores of 1.42 and -1.78 and $\mathrm{TM}$ values of $0.54 \pm 0.15$ and $0.50 \pm 0.15$ respectively. To further identify the active sites of the enzymes and drug binding potential, these models were superimposed with published crystal structures of structurally homologous enzymes co-crystallised with identified inhibitors. This included 4AYT (Shintre et al., 2013), an ATP-binding cassette (ABC) transporter found in the innermembrane of mitochondria and 6IPB (Zhang et al., 2020), a UDP-glucuronosyltransferase from Homo sapiens. The \% identity of these sequences with the corresponding A. pegreffii sequences were $35.47 \%$ and $23.03 \%$ respectively. The ABCB9 inhibitor binding site includes residues Tyr-504, Arg-507, Thr-506, Asp-291, Gln578, Glu-659, Ser-537, Gly-535, Ser-534, Gly-533, Ser-532, Lys-536, Ser-538, Ile-512. It is shown in Fig. 5B bound to the ATP nucleotide analogue phosphomethylphosphonic acid adenylate ester (ACP) that was observed in 4AYT. Overall, this represents an $85.7 \%$ sequence identity between active site domains. The active site residues of UGT3 fall within $4 \AA$ the inhibitory tartrate (TTA) molecule observed in 6IPB and includes Ala-309, Phe-310, Gly-311, Asn-312, His-313, Gln-360, Gly-374, His-375, Ala-376, Gly-377, Leu378, Lys-379, Ser-380, Met-394, Gln-400 (Fig. 5D). Active site identity remains low, (approx. 28.5\%), however TTA in both structures makes identical hydrogen bond interaction between the carboxylates moieties and main chain amides of each protein. In fact, few of the observed differences we suspect would preclude TTA binding in our model. For the aspartic protease ASP6, three possible models were produced, however the selected closest structural homologue model had a poor resolution with a C-score of 0.61 , a TM value of $0.80 \pm 0.09$, a RMSD of $3.0 \pm 2.2 \AA$, and normalized z-scores less than 3.48 (Fig. 5E). The protein structure for the ASP6 does not contain the second domain present in the glycoprotein of 6ROW (Scarff et al., 2020) from Haemonchus contortus (45.83\% identity). The catalytic residues were in part identified via structural comparison with human Progastricsin (1HTR; Moore et al., 1995) and ASP6 and include Gly-53, Thr-54, Ser-55, Phe-56, Asp-71 (Fig. 5F). The selected model for LKHA4 (E.C. 3.3.2.6) had a C-score of 1.42, a TM value of $0.91 \pm 0.06$ (Fig. 5G) and was modelled after 4GAA (Stsiapanava et al., 2014), a leukotriene A4 hydrolase from Xenopus laevis, a bifunctional zinc metalloenzyme co-crystallized with the inhibitor bestatin (BES). Overall, the enzyme shares a $39.09 \%$ identity with the A. pegreffii, however the active site residues of LKHA4 that fall within $4 \AA$ of BES in our model and the $\mathrm{Zn}^{2+}$ binding region shares a 70.5\% identity with 4GAA (Fig. 5H). They include Trp313, Met316, Glu320, Tyr388, His301, His297, Leu294, Glu298, Arg560, Leu269, Gly270, Gly271, Met272, Glu273, Phe135, Pro138 and Gln137. For CNDP2 (E.C. 3.4.13.18), a model was produced with a C-score of 1.87, a TM value of $0.98 \pm 0.05$ (Fig. 5I). The protein structure for the CNDP2 corresponds to 2ZOF (Unno et al., 2008), a carnosinase complexed with $\mathrm{Mn}^{2+}$ and a non-hydolyzable substrate analogue bestatin (BES) from Mus musculus. The enzyme shares a $54.62 \%$ identity with the $A$. pegreffii sequence overall, while the identity of the active site surrounding Bestatin shares a $73.6 \%$ identity. The residues of CNDP2 that fall within $4 \AA$ of the inhibitor include Asp134, His-101, Glu-168, Glu-169, Gly171, His-446, Thr-199, Asp-197, Thr-198, Gln-208, Glu-415, Ile-211, Gly-417, Ser-418, Ile419, Pro420, His-381, Met-214, Arg-344 and His381 (Fig. 5J). 


\section{Discussion}

Parasites have a myriad of survival strategies to warrant successful infection and propagation/reproduction within the hosts, being expressed through a highly specific genetic adaptations (Qu et al., 2019). Their genomes show great variability in genome size and organization due to the expansions of non-coding elements, such as long terminal repeat transposons and parasite specific gene families (Coghlan et al, 2019). Despite this, the free-living nematode Caenorhabditis elegans still remains the most studied nematode today, building the basis of our understanding of nematode physiology. Functionally, specific gene families in parasites reflect their biology, frequently encoding for proteases/peptidases, protease inhibitors, SCP/TAPS proteins (sperm-coating protein/Tpx-1/Ag5/PR-1/Sc7, from the cysteine-rich secretory protein superfamily), acetylcholinesterases, sensory receptors (G coupled receptors, GPCRs), cuticle maintenance proteins, fatty acid and retinol binding (FAR) proteins, glycosly transferases, chondroitin hydrolase, ABC transporters and kinases, as well as other taxa-restricted gene families involved in niche colonisation and immunity-evasion (Viney, 2018; Coghlan et al, 2019). However, the knowledge on pathways engaged in the active migration of infective larval stages through the host in respect to larvae failing to infect, and the array of responses when larvae face evolutionary-distant host, is mostly fragmented or unsolved for non-model parasites, although it has a practical importance in combating parasitosis.

\section{How larvae react to good (paratenic) vs bad (accidental) host?}

The striking difference between two transcriptome profiles of infecting Anisakis pegreffii larvae was largely attributed to host specificity, i.e. larvae infecting the rat (an accidental and evolutionary-distant host) and the fish (a paratenic and evolutionary-familiar host). Less different were profiles in respect to tissues of L3 migration (e.g. stomach, intestine, muscle), or to the designated larval stage (i.e. non-migrating, migrating, post-migrating, spiralised). Expectedly, L3 collected as dormant and spiralised stages from the fish visceral cavity showed profiles divergent from those infecting rat and, to a lesser extent fish, confirming that Anisakis spp. persist in paratenic host without essential development and growth (paratenesis) (Beaver 1969). Such evolutionary strategy allows multiple number of encounters between paratenic host and parasite, and consequently, the accumulation and extended lifespan of the latter (Anderson 1982).

Specific life history traits of Anisakis are hard to parallel in respect to other parasitic nematodes, creating a gap in knowledge transfer from well-studied and defined laboratory models. Family Anisakidae diverged from its terrestrial sister group Ascarididae approximately 150-250 Ma, but the split from their common ancestral host, a terrestrial amniote, happened already in Early Carboniferous (360.47 Ma). Anisakids acquired a semiaquatic tetrapod host, and as a result of lateral host-switches in Cenozoic, colonised marine mammals and co-evolved with their "new hosts". Therefore, the most closest referent system is believed to be the intestinal Ascaris genus; Ascaris lumbricoides that infects humans and causes ascariasis and A. suum in pigs (Wang, 2014) [although these are considered a single species based on morphological and genetic similarity, corroborated by the account of cross-infections between humans and pigs (Leles et al, 2012)]. A. suum exerts one of the most complex life cycles in its single host; ingested Ascaris eggs hatch and the infective L3 larvae undergo an extensive hepatotracheal migration, subsequently returning into the small intestine to reach adult stage. Such behavior somewhat resembles Anisakis L3 larvae infecting humans (accidental host), but greatly contrasts the infection pathway in marine mammal, their final host, where no migration occurs. While in A. summ each migratory stage exhibits strictly controlled spatiotemporal gene expression patterns, the most abundant transcripts per each stage are shared among stages and can be categorized in three common molecular function GO terms - binding (GO: 0005488; small molecule binding, protein binding, nucleic acid binding and ion binding), structural molecule activity (GO: 0005198; ribosome structural constituent, cuticle synthesis) and catalytic activity (GO: 0003824; hydrolase, oxidoreductase). This coincides well with GOs observed in A. pegreffii infecting evolutionary-distant rat and 
contrasts the observation in the evolutionary-familiar fish described herein. This transcriptomic synergy in A. summ and $A$. pegreffii larvae is further confirmed by two top represented KEGG pathways in both parasites; i.e. the Ribosome and Oxidative phosphorylation, the former utterly absent in A. pegreffii infecting fish.

A high transcription of ribosome-related genes during infection in homeotherm host reflects the surge in demand for proteins. Whether those involved in building up of larval cellular elements and cell division during growth towards L4 stage, or those necessary for enzymatic reactions related to increased energetic demands and production of essential excretory/ secretory products, can be scrutinised from downstream pathways. The ribosome production during the physiological cell cycle in higher eukaryotes starts at the end of mitosis, increases during G1, is maximal in G2 and stops during prophase (Leung et al., 2004), therefore upregulated Ribosome confirms ongoing of cell proliferation and growth in larval $A$. pegreffii infecting rat. The fact that this is observed only in the accidental hosts where larvae attempt to mature, but failing to reach an adequate attachment niche keep migrating, suggests a higher energetic and metabolic burden imposed on the larvae, eventually resulting in spent Anisakis. However, elements involved in the molting, such as structural constituents of cuticle, different collagens and cuticlins, feature the list of differentially expressed genes in larvae infecting both rat and sea bass, with the distinction that those in rat have been increasingly upregulated, while in the sea bass, some were even strongly downregulated. As production of cuticle constituents is stage- and molt cycle-specific and precedes the molting by hours in $C$. elegans (Page and Johnston, 2007) or possibly even days in A. suum (Wang, 2014), we hypothesize that $A$. pegreffii larvae are in the initial stages of their molt process, more advanced in a rat than in a seabass. This is corroborated by the fact that some of key enzymes for collagen assembly did not show differential regulation in any host, such as prolyl-4 hydroxylase (P4H). However, protein disulfide isomerase 2 (PDI-2), essential for embryonic development, proper molting, extracellular matrix formation and normal function of P4H in nematodes (Winter et al, 2007; 2013), was found slightly upregulated in rat-infecting larvae.

According to other most represented KEGG pathways, biosynthesis of secondary metabolites and oxidative phosphorylation were positively perturbed in both hosts, although evidently stronger in rat, which reflects the need to satisfy various metabolic needs and increased demand for energy-rich molecules, such as ATP. Glycolysis/ gluconeogenesis-associated DEGs were noted in larvae from both hosts with fatty acid metabolism significant only for those infecting rat. This confirms that carbohydrate metabolism is constitutively the essential energy source for infecting larvae, but the fatty and amino acid metabolisms are required for infection of homeotherm host. Similarly, A. suum L3 found in lung also show a prominent upregulation of lipid/ fatty acid metabolism that eventually decreases with the transition to L4 and adults (Wang, 2014). Conversely, quiescent non-feeding L3 of the strongylid Haemonchus contortus that completes its lifecycle in the abomasum of ruminants, depend mainly on stored lipid reserves to survive adverse conditions in the pastures before reaching its next host (Laing et al, 2013). Fascinating studies from C. elegans confirm that monosaturated lipids regulate fat accumulation and longevity, saturated fatty acid acclimation to temperature, PUFAs are required for growth, reproduction, neurotransmission, and as precursors for signaling molecules (Watts and Ristow, 2017), indicating how complex these pathways are. Nonetheless, we suggest that the similarities between the profiles triggered in $A$. suum pig infection and $A$. pegreffii rat infection are likely correlated to homeothermy of the accidental host.

Upregulated elements of KEGG Microbial metabolism in diverse environments only in Anisakis ratinfecting larvae further supports the effort of the larvae to adapt to environmental and metabolic changes and survive stress conditions in the homeotherm host. This pattern consists of different metabolic processes, such as carbohydrate, carbon fixation, methane, nitrogen, sulphur, amino acid metabolism, as well as metabolism of cofactors and vitamins, and xenobiotic degradation effectuated by bacteria (https://www.genome.jp/kegg-bin/show_pathway?map01120), being usually expressed in response to the heat shock (Tripathy et al., 2014). Noteworthy is that the most upregulated element of this pathway in both hosts was mitochondrial glutamate dehydrogenase, a crucial enzyme linking nitrogen and carbon metabolism where ammonia is either assimilated to provide glutamate as nitrogen storage molecule, or 
dissimilated to alpha-ketoglutarate for the tricarboxylic acid (TCA) metabolism. Unfortunately, while its role to provide of reducing equivalents in form of NADPH required for downstream redox reactions essential in Plasmodium falciparum antioxidant machinery has been rebutted (Storm et al., 2011), scarce information exist for its function in helminths. More precisely, structural models give no tangible implication for its functional role in host infection, being also dismissed as unsuitable as a drug target (Brown et al., 2014). However, another highly expressed transcript during rat infection listed within this KEGG was enolase. It is a multifunctional glycolytic enzyme found engaged in adhesion and invasion of intracellular apicomplexan Cryptosporidium parvum (Mi et al., 2017), as well as activation of fybrinolytic agent plasmin in Schistosoma mansoni intravascular life stages, where apparently helps the trematode to maintain anti-coagulated environment (Figueiredo et al, 2015). Similarly, nematode Trichinella spiralis enolase binds the host's plasminogen to activate the fibrinolytic system, degrades the extracellular matrix and promotes larval penetration of the tissue barrier during invasion (Jiang et al., 2019), which could be speculated for Anisakis enolase as well.

In spite of the evident cues for cell proliferation in rat-infecting L3 indicative of molting toward L4 stage, less represented KEGG pathways show downreagulation in both Cell senescence and Cell cycle. This apparently conflicting status; i.e. the blocking of transcripts programmed to arrests cell proliferation in response to different damaging stimuli (Muñoz-Espín Serrano 2014; Zumerle and Alimonti, 2020), contrasted by the blocking of transcripts that should support the cell cycle, could add to two likely coupled strategies. Firstly, KEGG Cell senescence and Cell cycle show similar levels of downregulation, implying that both processes are balanced, acting mutually in a feed-back loop. Being tightly coupled to cell growth, the efficient ribosynthesis enabled by high transcription rate of rDNA genes and the activity of ribosomal polymerases, allows a rapid cell proliferation required to meet cellular needs for ribosomes. However, under the stress conditions that affect the cell cycle and intracellular energy status (e.g. lack of nutrients), change in ribosynthesis is one of the cell strategies to retrieve homeostasis (Sengupta et al., 2010), likely to manifest in this case. Secondly, the homeothermic host environment, although offers the initial cues for growth, moulting and reproduction of A. pegreffii L3, possess additional conditions acting upon Anisakis' further development, which consequently result in parasites failure to survive. We can only speculate whether rat microbiome additionally contributes to such outcome, as in general microbiome interaction between the host and parasite differs for each specific case (Zaiss and Harris, 2016). However, it is logical to assume that Anisakis evolutionary has not been in contact with a terrestrial, homeotherm microbiome, which is likely to impose additional pressure on the larval survivor in the accidental host.

From the physiological stimuli-reaction standpoint, we can also hypothesise that the fate of the larval Anisakis in the accidental host is simply a result of larval exhaustion of nutrients and energy, spent during undetermined migration towards a niche that does not prove to be adequate, and stimulated by elevated host's temperature. In contrast, in the parathenic exothermic host where metabolic pathways are moderately upregulated or silenced, larvae prepare for paratenesis that warrants their survival. This is inferred through FoxO signalling pathway, which was substantially downregulated in the accidental and upregulated in the paratenic host. While the pathway encompasses transcription factors that regulate expression of many downstream genes involved in cellular processes such as apoptosis, cell-cycle control, glucose metabolism, oxidative stress resistance, and longevity (Tia et al., 2018), the highest upregulated transcript (more then 10fold) in sea bass-infecting Anisakis is gamma-aminobutyric acid receptor-associated protein (GABARAP), recognised as a hallmark for autophagy (Oshumi, 2014), but also encompassed within KEGG Longevity regulating pathway. GABARAP accumulates in the pericentriolar material under nutrient rich conditions, from which is translocated during starvation to form autophagosomes (Joachim et al., 2017). Although FoxO-GABARAP axis has been studied in colorectal and ovarian cancers, it is tempting to speculate that the autophagy in fish-infecting larvae represents a safety mechanism for their successful survival. Namely, in cancer FoxO3a senses variation in AMP/ATP ratio by decreased gycolysis, which activates FoxO3a transcriptional program, resulting in activation of genes involved in autophagic flux, namely GABARAP, GABARAPL1, GABARAPL2 and MAP1LC3 (Grossi et al., 2019). In general, autophagy is an essential 
cellular mechanism that enables the cell to counter-balance various demands by producing autophagosomes that engulf a wide range of intracellular material and transport it to lysosomes for subsequent degradation (Nakatogawa, 2020). While the basal autophagy acts as a housekeeping mechanism, the inducible autophagy starts by engulfment of bulk cytoplasm in times of stress, such as nutrient deprivation. Although this process still needs to be characterised in parasitic helminths, $C$. elegans employs it to remove aggregate-prone proteins, paternal mitochondria, spermatid-specific membranous organelles; remodeling during dauer development; degradation of the miRNA-induced silencing complex; synapse formation and in the germ line; to promote the stem cell proliferation; removal of apoptotic cell corpses; lipid homeostasis and in the ageing process (Palmisanoa and Meléndez, 2018). We suggest that in Anisakis larvae during exothermic conditions of infection, GABARAP induced through FoxO and/or Longevity regulating pathway, triggers autophagy (KEGG Autophagy-animal, -yeast, -other) that eventually balances the metabolic rate in larvae by clearing damaged/used organelles to prepare the nematode for indefinite paratenesis, necessary to counteract larval ageing and death. To further support this, KEGG Longevity regulating pathway showed to be upregulated in larvae infecting seabass and downreagulated in those infecting a rat. The relationship between Anisakis autophagy, metabolic balancing and longevity was supported by one of the highly expressed elements of KEGG Longevity regulating pathway - adiponectin receptor preotein 2; one of two transmembrane receptors that bind and activate adiponektin in humans, regulating glucose and lipid metabolism (Buechler et al., 2010). As the calorie restriction and consequent limited buildup of toxic cellular waste has been shown to extend the lifespan in a range of organisms (Mannack and Lane, 2015), it seems that the same strategy could be employed for Anisakis paratenesis.

\section{Anisakis pegreffii virulence factors and their potential drug-targeting}

We defined $A$. pegreffii virulence factors as those transcripts expressing the highest upregulation common for A. pegreffii migrating through both hosts in respect to larvae that failed to do so. From the initial 65 transcripts, we selected 35 that showed at least two-fold change expressed in at least one of the host (Table 3), and then discarded constitutive cuticle elements and those with no annotation. That left us with several catalysts and transporters, some being recognised as excretory/ secretory (ES) products; cytosolic non-specific dipeptidase (CNDP2), leukotriene A-4 hydrolase (LKHA4), aspartic protease 6 (ASP6), ATP-binding cassette sub-family B member 9 (ABCB9), and UDP-glucuronosyltransferase (UGT3). Some of them (CNDP2, ASP6 and ABCB9) have subfamily/ subgroup members identified as surface-exposed molecules on the extracellular vesicles (EVs) of the trematode Fasciola hepatica (de la Torre-Escudero et al., 2018). EVs have been recognised as essential mediators of communication (through molecular signals such as proteins, lipids, complex carbohydrates, mRNA, microRNA and other non-coding RNA species) between parasite and host, particularly in helminth immunomodulatory strategy, suggesting that these transcripts might have a role in Anisakis-host cell signaling. Interestingly, none have been identified in previous works (Kim et al., 2018; Llorens et al., 2018), probably because those focused on transcriptomic profiles of dormant, non-infecting or in vitro cultured larvae. Specific temporal regulation of certain virulence factors might also depend on their other putative functions and co-expression networks. For instance, hyaluronidase, a hydrolytic enzyme that degrades the glycosaminoglycan hyaluronic acid, erected as crucial for pathogenesis of Ancylostoma caninum, Anisakis simplex and A. suum (Hotez et al, 1994; Wang, 2014; Ebner et al, 2018) showed no differential regulation in either of hosts studied herein, albeit expressed in the transcriptome. Rhoads et al. (2001) noted its release in A. suum when transitioning from L3 to L4, corroborating its other important functions next to facilitation of larval penetration, such as feeding, proper molting and development. If this is also true for Anisakis, we might have missed the point when the need for this enzyme surges.

The versatile gene content and unequal protein family representation among nematodes and other helminths has been established and reflects uniqueness of parasite biology and different pathogenic strategies (Coghlan et al, 2019). It is also the result of specificities of each host-parasite relationship formed during their evolution. In order to investigate whether virulence factors erected for A. pegreffii are shared between other 
helminths and investigate their potential for repurposing of existing therapeutics, we have performed orthology-directed phylogenetic analyses of A. pegreffii ABCB9, UGT3, ASP6, LKHA4 and CNDP2 to visualize gene gain and loss among representative helminths (Figure 4). We have also determined and identified the 3-dimensional structures and catalytic sites for all five above mentioned DEGs utilizing online modelling techniques and comparing our models to structures co-crystalized with inhibitors or substrate analogues (Figure 5).

The results of our phylogenetic analyses support the premise that the four families (ABCB9s, ASP6s, LKHA4s and CNDP2s) are present in almost all Nematoda, platyhelminthes and metazoans examined, except for the Trematoda, Cestoda and vertebrate species that have lost UGT3. The high levels of duplication and wide-spread occurrence of all five target genes in closely related T. canis, A. suum, $P$. univalens and also $H$. contortus, suggests that these genes may have vital biological functions as virulence factors in these extant species. However, it is not clear why the Trematoda, Cestoda and outgroup species examined do not contain any recognizable UGT3 gene, but leads us to propose that these observations may be due to substantial divergence of UGT3 or incorporation of protein domains by horizontal gene transfer that has not been detected in this study.

Aspartic protease 6 is an endopeptidase involved in haemoglobin digestion, tissue penetration or host-derived nutrient digestion in helminths (Koehler et al., 2007; Ebner et al. 2018). The importance of proteases and protease inhibitors for helminths is reflected in their vast representation in nematode and platyhelminth species, as noted in a large comparative genomic study of parasitic worms (Coghlan et al, 2019). Aspartic proteases have been found especially abundant in Clade IV and V Nematoda, which is in general agreement with our phylogenetic inference of orthologues. Asp6 is one of the first transcripts upregulated in Ascaris suum upon contact with porcine epithelial cells (Ebner et al, 2018) and an altered homologue of aspartic protease 1 from Necator americanus has been selected as a target for human hookworm vaccine development (Hotez et al, 2013). Aspartic protease 6 has also been targeted in trypanosamotids therapy (causative agents of leishmaniasis, Chagas' disease and sleeping sickness) by canonical (DAN, EPNP, pepstatin A) and anti-HIV aspartic peptidase inhibitors (amprenavir, indinavir, lopinavir, nelfinavir, ritonavir and saquinavir). The latter inhibitors affected parasite's homeostasis, through elevated production of reactive oxygen species, apoptosis, loss of the motility and arrest of proliferation/growth (Santos et al., 2013). Drugbank lists several experimental chemotherapeutics targeting aspartic proteases of malarian parasite Plasmodium falciparum (artenimol) and fungus Candida albicans (ethylaminobenzylmethylcarbonyl, 1-amino-1-carbonyl pentane, butylamine, 1-hydroxy-2-amino-3cyclohexylpropane, 4-methylpiperazin-1-Yl carbonyl), as well as other human targets with aspartic-type endopeptidase activity involved in other pathogenesis, such as in Alzheimer's disease (https://go.drugbank.com).

ATP-binding cassette sub-family B member 9 belongs to a large group of multidrug resistance (MDR)/ transporters associated with antigen processing (TAP) transmembrane proteins. ABCB9 proteins are known therapeutic targets in disease (Shintre 2013) and well known to bind and confer drug resistance in cancer cells (Jin 2012). Located in lysosomal membrane, they use ATP-generated energy to translocate cytosolic peptides to the lysosome for processing (Zhao et al., 2008). However, until their functional characterisation in helminths, we cannot state whether the protein is lysosomal, or rather involved in the efflux of chemically unchanged organic xenobiotics, as some of ABCB members. The efficiency of in vitro anisakiasis treatment by inhibitors of other MDR members (ABCB/P-glycoprotein and MRX1) proved to be efficient (Mladineo et al., 2017), but no treatments targeting ABCB9 so far have been reported (https://go.drugbank.com). A genome-wide identification of ABC transporters in monogeneans identified orthologues of ABCB family in Gyrodactylus salaris, Protopolystoma xenopodis, Neobenedenia melleni, and specifically ABCB9 in Eudiplozoon nipponicum, as well as C. elegans (Caña-Bozada et al., 2019). This is in general agreement with our orthology inference, except for the presence of orthologues of ABCB9 in G. salaris, which might be the result of stringent criteria we used for orthologue identification. In general, ABC transporters show independent losses and expansions within parasitic worms (Coghlan et al, 2019). 
Cytosolic non-specific dipeptidase, also known as carboxypeptidase of glutamate-like (CPGL), catalyses the hydrolysis of peptides, being found significantly upregulated in adult stages of a tapeworm Taenia pisiformis, presumably associated to amino-acid transport and metabolism (Zhang, 2019). However, human CNDP2 figures as an important tumor suppressors in gastric, hepatocellular and pancreatic cancers that inhibits cell proliferation, and induces apoptosis and cell cycle arrest, via activation of mitogenactivated protein kinase (MAPK) pathway (Zhang et al., 2013). It is tempting to speculate whether Anisakis CNDP2 also serves in MAPK pathway, the latter employed in cell communication during helminth development and homeostasis (Dissous et al., 2006). No treatments targeting CNDP2 so far have been reported in Drugbank (https://go.drugbank.com).

Leukotriene A4 hydrolase has been studied as ESP of Schistosoma japonicum. The trematode synthesise proinflammatory mediators prostaglandins through arachidonic-acid metabolism that uses lecithin to generate arachidonate, converts it in leukotriene A4 and then more stable leukotriene B4 by leukotriene A4 hydrolase (The Schistosoma japonicum Genome Sequencing and Functional Analyses Consortium, 2009). Interestingly, prostaglandins and leukotrienes contribute to metabolism or maturation of the organism and communication with the host on a cellular basis, acting as immunomodulators and eosinophil attractants (Noverr et al, 2003). In S. japonicum they have been suggested to induce chemokine-receptor-mediated cell migration and leukocyte migration into inflamed tissue, which for the parasite is essential for survival as it promotes granuloma formation around expelled eggs. How Anisakis larvae benefit from stimulation of proinflammatory host reaction through upregulation of leukotriene A4 hydrolase is not clear, as inflammation favors propagation of only few parasites (Sorci and Faivre, 2009). Of 22 investigated drugs targeting human LKHA4, only captopril has been approved as an inhibitor of angiotensin-converting enzyme in regulation of blood pressure, having also an unknown pharamcological action on LKHA4 (https://go.drugbank.com).

Anisakis UDP-glucuronosyltransferase (UGT) homologues were significantly upregulated in larvae penetrating epithelial barriers of both hosts types in our study (UGT47, UGT50, UGT58), and a couple more reconstructed in the transcriptome were classified as DEGs only in rat (upregulated UGT50 and downregulated UGT60). UDP-glucuronosyltransferases catalyze glucuronidation reaction, the addition of polar glucuronic acid to lipophilic substrates promoting their elimination and clearance from the organism. As such they are important part of detoxification process and have been associated with benzimidazole resistance phenotype in $H$. contortus (Matoušková et al, 2018) or naphtalophos biotransformation (Kotze et al., 2014). Thirty four UGTs were reconstructed in the transcriptome of $H$. contortus (Laing et al, 2013), with four of these are putative orthologues to A. pegreffii UGT3 (Figure 4). According to KEGG orthology, UGTs interlink various metabolic pathways, such as Pentose and glucuronate interconversions, Ascorbate and aldarate metabolism, Steroid hormone biosynthesis, Retinol metabolism, Porphyrin and chlorophyll metabolism, Metabolism of xenobiotics by cytochrome P, Drug metabolism, Biosynthesis of secondary metabolites. Because it is essential for adult worm survival and due to its large, easily-targeted extracellular domain, in Brugia malayi it was selected as a potential drug target for lymphatic filariasis (Flynn et al, 2019). Two compounds targeting UGT3 are experimental and/or under investigation; kaempherol and quercetin, both flavonols with unknown pharmacological action and antioxidant properties. The latter however, has many targets in addition to UGT3, but it mainly inhibits quinone reductase 2 of the $P$. falciparum causing the lethal oxidative stress (https://go.drugbank.com). Interestingly, UGTs are also known for mediating metabolic inactivation of lipophilic cancer drugs. However, recently has been suggested that dysregulation of UGT expression might promote oncogenic pathways by metabolizing endogenous molecules such as steroids and bioactive lipids and disturbing homeostasis (Allain et al, 2020). The versatile role of these enzymes and detoxification processes is also observed in dauer C. elegans larvae where it has been proposed that cytochrome P450, UDP-glucuronosyltransferase and glutathione S-transferase perform vital clearance of toxic lipophilic and electrophilic metabolites associated with ageing and reduced longevity (McElwee et al, 2004). In study performed by Rausch et al (2018), UGTs and glutathione S-transferases were differentially regulated between Heligmosomoides polygyrus infecting germfree and conventional 
specific pathogen-free mice, suggesting that these detoxification enzymes also participate in nematode sensing of its microbial environment. Host microbiome has been demonstrated as important factor in shaping host-parasite relationships (Zaiss and Harris, 2016); furthermore H. polygyrus showed reduced fitness in germfree mice (Rausch et al, 2018). This represents an axis of investigation that must be explored in the future for A. pegreffii and might prove to be the missing explanatory link behind its success or failure to infect evolutionary distant hosts.

The discovery of new anthelmintic drug targets with broad-spectrum efficacy is expensive and time consuming. At present, approximately \$2.6USD billion in direct costs and 10-15 years is the average length of time required to progress from the concept of a new therapy or drug target to a new molecular entity on the market Tamimi and Ellis. 2009). In addition, the rate of success is less than 5\%, with less than $20 \%$ of compounds entering clinical trials actually receiving FDA approval over this time (Kola and Landis 2004; DiMasi et al., 2010). Our tertiary structure predictions and modelling analyses present the bases for the repurposing of selected candidate and currently available inhibitor molecules that should be incorporated in future investigations and may provide broad-spectrum efficacy particularly for all Clade III and V nematodes examined. For instance, BES is a well-known dipeptidase inhibitor for the aforementioned enzyme classes 3.3.2.6 and 3.4.13.18 (Andberg et al., 2000; Lenney 1990), while the ATP nucleotide analogue known as ACP would most likely bind ABCB9 due to the highly homologous nature of the active sites. Overall, inhibition may be possible with these candidate molecules, but more importantly targeted drug discovery efforts that could produce highly selective nematode species-specific inhibitors, might benefit from utilizing the drug sites modelled herein.

\section{Figure Legends}

Figure 1. Sample grouping according to gene expression variation in collected Anisakis pegreffii L3 larvae during experimental infections of a paratenic and an accidental host, Dicentrarchus labrax and Rattus norvegicus, respectively. Raw counts were normalized by variance stabilizing transformation and profiles are outlined using principal component analyses (PCA).

Figure 2. Venn diagram showing overlap between three groups of differentially expressed genes (DEGs) identified in Anisakis pegreffii L3 larvae during experimental infections: Migrating vs. non-migrating larvae in rat Rattus norvegicus, migrating vs. non-migrating larvae in seabass Dicentrarchus labrax and the interaction: larvae showing different regulation in two hosts. All larvae that successfully penetrated host mucosal barriers were considered migrating and compared against all those that remained inside gastrointestinal system of the same host.

Figure 3. Frequency distribution of differentially expressed genes (DEGs) in top 10 KEGG metabolic and signaling pathways identified in Anisakis pegreffii L3 larvae during experimental infections of a paratenic and an accidental host, Dicentrarchus labrax and Rattus norvegicus, respectively.

Figure 4. Phylogenetic species-tree reconstruction of homologous gene families corresponding to the five DEGs of interest across 28 species. The consensus tree is based on losses and gains of orthologous groups corresponding to the protein sequence alignments of the five target DEGs of Anisakis pegreffii: ABCB9, UGT3, ASP6, LKHA4 and CNDP2 (labelled with*). Only protein sequences that comply within the boundaries of the stringent sequence similarity cut-offs, E.C., GO and InterPro terms and/or descriptions are depicted. The numbers in colored circles represent the total number of gene families corresponding to a particular gene based on orthology. A missing value indicates the absence of an orthologous group 
corresponding to any of the five target DEGs of A. pegreffii. Bioproject GenBank accession numbers are provided (in parentheses) for all reference sequences.

Figure 5. Predicted tertiary structures of the selected drug therapy targets. The analysed DEGs include: AB, ATP-binding cassette sub-family B member 9 (ABCB9); C-D, UDP-glucuronosyltransferase (UGT3); EF, aspartic protease 6 (ASP6); G-H, leukotriene A-4 hydrolase (LKHA4); I-J, cytosolic non-specific dipeptidase (CNDP2). Predicted tertiary structures of the five DEG monomers are shown to the right. Locations of the $\mathrm{C}$ - and N-terminus in the predicted tertiary structures of each DEG are labelled. The predicted tertiary structure of the ABCB9 (A) showing the location of the active site in salmon (B) and phosphomethylphosphonic acid adenylate ester (ACP) used as an inhibitor (green). The UGT3 predicted tertiary structure (C) with the location of the active site shown in purple (D) and inhibitory tartrate (TTA) in light blue. The ASP6 predicted tertiary structure (E) showing the location of the active site (F) in light blue. The leukotriene A-4 hydrolase (LKHA4) predicted tertiary structure $(\mathrm{G})$ showing the location of the active site shown in green $(\mathrm{H})$ and inhibitory bestatin (BES) in yellow as well as the $\mathrm{Zn}^{2+}$ binding region. The cytosolic non-specific dipeptidase (CNDP2) predicted tertiary structure (I) showing the location of the active site shown in green $(\mathrm{J})$ and inhibitory bestatin (BES) shown in salmon.

Figure 6. Schematic representation of the fate of Anisakis pegreffii L3 larvae after infecting evolutionaryfamiliar, parathenic host seabass (Dicentrarchus labrax) and evolutionary-distant, accidental host rat (Rattus norvegicus).

\section{Table Legends}

Table 1. Summary of the design and outcome of experimental infections of Dicentrarchus labrax and Rattus norvegicus with Anisakis pegreffii L3 larvae. Number of intubated animals, sampling time, minimum, maximum and median percent (\%) recovery rate, larval phenotype and site of collection within the host are shown.

Table 2. Statistics of Anisakis pegreffii de novo assembly and annotation summary.

Table 3. Differentially expressed genes (DEGs) identified in Anisakis pegreffii L3 migrating vs nonmigrating larvae during experimental infections of a paratenic and an accidental host, Dicentrarchus labrax and Rattus norvegicus, respectively. Top 10 DEGs show different regulation in the two hosts and bottom 35 show congruent profiles for both hosts and at least two-fold change in expression in one of the hosts. Putative virulence factors selected as potential drug targets are depicted in bold. DEGs were identified at Benjamini-Hochberg false discovery rate $(\mathrm{FDR})<0.05$.

\section{Supplementary material}


Supplementary Figure 1. Frequency distribution of differentially expressed genes (DEGs) in top 10 Gene Ontology terms in Biological process, Molecular function and Cellular component identified in Anisakis pegreffii L3 larvae during experimental infections of a paratenic and an accidental host, Dicentrarchus labrax and Rattus norvegicus, respectively.

Supplementary Table 1. Sample description and formation of RNAseq libraries.

Supplementary Table 2. The reference assembled transcriptome of Ansiakis pegreffii with nucleotide and amino acid sequences and functional annotation.

Supplementary Table 3. Gene-level raw counts of each RNAseq library used for differential expression analyses of Anisakis pegreffii L3 larvae during experimental infections of Dicentrarchus labrax and Rattus norvegicus.

Supplementary Table 4. Complete list of differentially expressed genes (DEGs) identified in Anisakis pegreffii L3 larvae during experimental infections: Migrating vs. non-migrating larvae in rat Rattus norvegicus, migrating vs. non-migrating larvae in seabass Dicentrarchus labrax and the interaction: larvae showing different regulation in two hosts.

Supplementary Table 5. Results of enrichment analyses of GO terms within sets of DEGs identified in Anisakis pegreffii L3 larvae during experimental infections: Migrating vs. non-migrating larvae in rat Rattus norvegicus, migrating vs. non-migrating larvae in seabass Dicentrarchus labrax and the interaction: larvae showing different regulation in two hosts. Goseq package for $\mathrm{R}$ was used for the analyses taking gene length bias into account.

Supplementary Table 6. Results of enrichment analyses of KEGG metabolic and signaling pathways via KEGG Orthologues (KO) within sets of DEGs identified in Anisakis pegreffii L3 larvae during experimental infections: Migrating vs. non-migrating larvae in rat Rattus norvegicus, migrating vs. non-migrating larvae in seabass Dicentrarchus labrax and the interaction: larvae showing different regulation in two hosts. Goseq package for $\mathrm{R}$ was used for the analyses taking gene length bias into account.

Supplementary Table 7. Complete list of orthologous protein families for each of the five DEGs of interest across 28 species including additional annotations.

\section{Acknowledgements}

High performance computing was conducted using the resources of computational cluster Isabella hosted by the University Computing Centre, University of Zagreb (SRCE), Croatia.

\section{Funding}


This research was funded by Croatian Scientific Foundation, grant numbers IP-2014-5576 (AnGEl: Anisakis spp.: Genomic Epidemiology) and IP-2018-01-8490 (AnisCar: Anisakis as a carcinogen: Daring to bust Lancet's myth or revealing its true colours) awarded to Ivona Mladineo.

\section{References}

Accelerated profile HMM searches. S. R. Eddy. PLOS Comp. Biol., 7:e1002195, 2011.

Alexander Dobin 1, Carrie A Davis, Felix Schlesinger, Jorg Drenkow, Chris Zaleski, Sonali Jha, Philippe Batut, Mark Chaisson, Thomas R Gingeras

Allain, EP, M Rouleau, E Lévesque, C Guillemette. 2020. Emerging roles for UDP-glucuronosyltransferases in drug resistance and cancer progression. British Journal of Cancer 122:1277-1287.

Andberg, M, A Wetterholm, JF Medina, JZ Haeggström. 2000. Leukotriene A4 hydrolase: a critical role of glutamic acid-296 for the binding of bestatin. Biochemical Journal 345:621-625.

Anderson R. Host-parasite relations and evolution of the Metastrongyloidea (Nematoda). Mem Mus Natl Hist Nat Ser A Zool. 1982;123:129-32.

Andrews S. (2018). FastQC: a quality control tool for high throughput sequence data. Available online at: http://www.bioinformatics.babraham.ac.uk/projects/fastqc

Audicana, M. T., \& Kennedy, M. W. (2008). Anisakis simplex: From obscure infectious worm to inducer of immune hypersensitivity. Clinical Microbiology Reviews, 21(2), 360-379. https://doi.org/10.1128/CMR.00012-07.

Baird FJ, Su X, Aibinu I, Nolan MJ, Sugiyama H, Otranto D, et al. The Anisakis transcriptome provides a resource for fundamental and applied studies on allergy-causing parasites. PLoS Negl Trop Dis. (2016) 10:e0004845. doi: 10.1371/journal.pntd.00 04845

Bao M, Pierce GJ, Pascual S, Gonza' lez-Muñoz M, Mattiucci S, Mladineo I, et al. Assessing the risk of an emerging zoonosis of worldwide concern: anisakiasis. Sci Rep. 2017; 7: 43699.

Bao M, Pierce GJ, Strachan NJC, Pascual S, González-Muñoz M, Levsen A. 2019. Human health, legislative and socioeconomic issues caused by the fish-borne zoonotic parasite Anisakis: Challenges in risk assessment. Trends in Food Science \& Technology 86, 298-310 doi.org/10.1016/j.tifs.2019.02.013

Beaver PC. The nature of visceral larva migrans. J Parasitol. 1969;55:3-12.

BioBam Bioinformatics. (2019). OmicsBox-Bioinformatics made easy (Version 1.4.11).

Bolger AM, Lohse M, Usadel B. Trimmomatic: a flexible trimmer for Illumina sequence data. Bioinformatics (2014) 30:2114-20. doi: 10.1093/bioinformatics/btu170

Bouwknegt M, Devleesschauwe B, Graham H, Robertson LJ, van der Giessen J. Prioritization of foodborne parasites in Europe. Euro Surveill. 2018; 23: 17-00161. https://doi.org/10.2807/1560-7917.ES. 2018.23.9.17-00161https://doi.org/10.2807/1560-7917.ES.2018.23.9.17-00161.

Bryant DM, Johnson K, DiTommaso T, Tickle T, Couger MB, Payzin-Dogru D, Lee TJ, Leigh ND, Kuo TH, Davis FG, Bateman J, Bryant S, Guzikowski AR, Tsai SL, Coyne S, Ye WW, Freeman RM Jr, Peshkin L, Tabin CJ, Regev A, Haas BJ, Whited JL. A Tissue-Mapped Axolotl De Novo Transcriptome Enables Identification of Limb Regeneration Factors. Cell Rep. 2017 Jan 17;18(3):762-776. doi: 10.1016/j.celrep.2016.12.063. PubMed PMID: 28099853; PubMed Central PMCID: PMC5419050.

Buchfink, B., Xie, C., \& Huson, D. H. (2015). Fast and sensitive protein alignment using DIAMOND. Nature methods, 12(1), 59-60.

Bus`elićI,TrumbićŽ,HrabarJ,VrbatovićA,Boc $\square$ inaI,MladineoI.Molecularandcellularresponseto experimental Anisakis pegreffii (Nematoda, Anisakidae) third-stage larval infection in rats. Front Immunol. 2018; https://doi.org/10.3389/fimmu.2018.02055.

C. Llorens, S. C. Arcos, L. Robertson, R. Ramos, R. Futami , B. Soriano, S. Ciordia , M. Careche , M. González-Muñoz, Y. Jiménez-Ruiz, N. Carballeda-Sangiao, I. Moneo , J. P. Albar , M. Blaxter, A. Navas 2018. Functional insights into the infective larval stage of Anisakis simplex s.s., Anisakis 
pegreffii and their hybrids based on gene expression patterns BMC Genomics (2018) 19:592 https://doi.org/10.1186/s12864-018-4970-9

Camacho, C., Coulouris, G., Avagyan, V., Ma, N., Papadopoulos, J., Bealer, K., \& Madden, T. L. (2009). BLAST+: architecture and applications. BMC bioinformatics, 10(1), 421.

Caña-Bozada, V, FN Morales-Serna, A García-Gasca, R Llera-Herrera, EJ Fajer-Ávila. 2019. Genome-wide identification of ABC transporters in monogeneans. Molecular and Biochemical Parasitology 234:111234.

Cavallero S, Lombardo F, Su X, Salvemini M, Cantacessi C, D'Amelio S. Tissue-specific transcriptomes of Anisakis simplex (sensu stricto) and Anisakis pegreffii reveal potential molecular mechanisms involved in pathogenicity. Parasit Vectors (2018) 11:31. doi: 10.1186/s13071-017- 2585-7

CD-HIT: accelerated for clustering the next generation sequencing data", Limin Fu, Beifang Niu, Zhengwei Zhu, Sitao Wu \& Weizhong Li. Bioinformatics, (2012) 28:3150-3152

Christa Buechler 1 , Josef Wanninger, Markus Neumeier 2010. Adiponectin receptor binding proteins-recent advances in elucidating adiponectin signalling pathways. FEBS Lett 584:4280-6. doi: 10.1016/j.febslet.2010.09.035.

Christiam Camacho 1, George Coulouris, Vahram Avagyan, Ning Ma, Jason Papadopoulos, Kevin Bealer, Thomas L Madden

Christiane Kruse Fæstea,*, Karen R. Jonscherb, Maaike M.W.B. Doopera, Wolfgang Egge-Jacobsenc, Anders Moenc, Alvaro Daschnerd, Eliann Egaasa, Uwe Christians 2014. Characterisation of potential novel allergens in the fish parasite Anisakis simplex. EuPA Open Proteomics 4 (2014) 140-155 doi.org/10.1016/j.euprot.2014.06.006

Cipriani, P., Acerra, V., Bellisario, B., Sbaraglia, G.L., Cheleschi, R., Nascetti, G., Mattiucci, S., 2016. Larval migration of the zoonotic parasite Anisakis pegreffii (Nematoda: Anisakidae) in European anchovy, Engraulis encrasicolus: implications to seafood safety. Food Control 59, 148-157.

Consortium, TU. 2018. UniProt: a worldwide hub of protein knowledge. Nucleic Acids Research 47:D506D515.

Corcuera MT, Rodr' 1guez-Bobada C, Zuloaga J, Go'mez-Aguado F, Rodr' 1guez-Perez R, Mendizabal A' , et al. Exploring tumourigenic potential of the parasite Anisakis: a pilot study. Parasitol Res. Parasi- tology Research; 2018; 117: 3127-3136. https://doi.org/10.1007/s00436-018-6008-2.

D’Amelio S, Mathiopoulos KD, Santos CP, Pugachev ON, Webb SC, Picanço M, et al. Genetic markers in ribosomal DNA for the identification of members of the genus Anisakis (Nematoda: Ascaridoidea) defined by polymerase-chain-reaction-based restriction fragment length polymorphism. Int J Parasitol. (2000) 30:223-6. doi: 10.1016/S0020-7519(99)0 0178-2

de la Torre-Escudero, E, JQ Gerlach, AP Bennett, K Cwiklinski, HL Jewhurst, KM Huson, L Joshi, M Kilcoyne, S O’Neill, JP Dalton. 2019. Surface molecules of extracellular vesicles secreted by the helminth pathogen Fasciola hepatica direct their internalisation by host cells. PLoS neglected tropical diseases 13:e0007087.

DiMasi, JA, L Feldman, A Seckler, A Wilson. 2010. Trends in risks associated with new drug development: success rates for investigational drugs. Clin Pharmacol Ther 87:272-277.

Dissous, C, N Khayath, J Vicogne, M Capron. 2006. Growth factor receptors in helminth parasites: Signalling and host-parasite relationships. FEBS Letters 580:2968-2975.

Dobin A, Davis CA, Schlesinger F, Drenkow J, Zaleski C, Jha S, et al. STAR: Ultrafast universal RNA-seq aligner. Bioinformatics (2013) 29:15-21. doi: 10.1093/bioinformatics/bts635

E. Łopien'ska-Biernat, Ł.Paukszto, Jan Paweł Jastrzebski, Kamil Myszczyn'ski, Iwona Polak, Robert Stryinski 2019. Genome-wide analysis of Anisakis simplex sensu lato: the role of carbo- hydrate metabolism genes in the parasite's development, International Journal for Parasitology, 49, 933-943. doi.org/10.1016/j.ijpara.2019.06.006 
Ebner F, Kuhring M, Radonic' A, Midha A, Renard BY and Hartmann S (2018) Silent Witness: DualSpecies Transcriptomics Reveals Epithelial Immunological Quiescence to Helminth Larval Encounter and Fostered Larval Development. Front. Immunol. 9:1868. doi: 10.3389/fimmu.2018.01868

Elz $\square$ bieta Lopieńska-Biernata, Isabel Medinab, Mónica Carrera 2019. Proteome profiling of L3 and L4 Anisakis simplex development stages by TMT-based quantitative proteomics Journal of Proteomics 201, 1-11 doi.org/10.1016/j.jprot.2019.04.006

Emms, D. M., \& Kelly, S. (2015). OrthoFinder: solving fundamental biases in whole genome comparisons dramatically improves orthogroup inference accuracy. Genome biology, 16(1), 157.

exp inf fish

Figueiredo BC, Da'dara AA, Oliveira SC, Skelly PJ (2015) Schistosomes Enhance Plasminogen Activation: The Role of Tegumental Enolase. PLoS Pathog 11(12): e1005335. doi:10.1371/journal.ppat.1005335

Flynn AF, Joyce MG, Taylor RT, Bennuru S, Lindrose AR, Sterling SL, et al. (2019) Intestinal UDPglucuronosyltransferase as a potential target for the treatment and prevention of lymphatic filariasis. PLoS Neg1 Trop Dis 13(9): e0007687. https://doi.org/10.1371/journal.pntd.0007687

Giancarlo Lancini, Rolando Lorenzetti 1993. Biosynthesis of Secondary Metabolites. In:biotechnology of Antibiotics and other bioactive microbial metabolites. Springer Science = Business Media, New York, pp. 95-132.

Grabherr MG, Haas BJ, Yassour M, Levin JZ, Thompson DA, Amit I, Adiconis X, Fan L, Raychowdhury R, Zeng Q, Chen Z, Mauceli E, Hacohen N, Gnirke A, Rhind N, di Palma F, Birren BW, Nusbaum C, Lindblad-Toh K, Friedman N, Regev A. Full-length transcriptome assembly from RNA-seq data without a reference genome. Nat Biotechnol. 2011 May 15;29(7):644-52. doi: 10.1038/nbt.1883. PubMed PMID: 21572440.

H. Wickham. ggplot2: Elegant Graphics for Data Analysis. Springer-Verlag New York, 2016.

Haarder S, Kania PW, Bahlool Q.Z.M., Buchmann K. 2013. Expression of immune relevant genes in rainbow trout following exposure to live Anisakis simplex larvae Experimental Parasitology 135:564569 doi:10.1016/j.exppara.2013.09.011

Haas BJ, Papanicolaou A, Yassour M, Grabherr M, Blood PD, Bowden J, Couger MB, Eccles D, Li B, Lieber M, Macmanes MD, Ott M, Orvis J, Pochet N, Strozzi F, Weeks N, Westerman R, William T, Dewey CN, Henschel R, Leduc RD, Friedman N, Regev A. De novo transcript sequence reconstruction from RNA-seq using the Trinity platform for reference generation and analysis. Nat Protoc. 2013 Aug;8(8):1494-512. Open Access in PMC doi: 10.1038/nprot.2013.084. Epub 2013 Jul 11. PubMed PMID:23845962.

Haemonchus contortus J. BioSci. Biotech. 2014, 3:49-60.

Hanbo Chen (2018). VennDiagram: Generate High-Resolution Venn and Euler Plots. R package version 1.6.20. https://CRAN.R-project.org/package=VennDiagram

Hitoshi Nakatogawa 2020 Mechanisms governing autophagosome biogenesis Nature Reviews Molecular Cell Biology 21, 439-458

Hochberg NSS, Hamer DHH. Anisakidosis: perils of the deep. Clin Infect Dis. 2010; 51: 806-812. https://doi.org/10.1086/656238.

Hotez P, Cappello M, Hawdon J, Beckers C, Sakanari J. Hyaluronidases of the gastrointestinal invasive nematodes Ancylostoma caninum and Anisakis simplex: possible functions in the pathogenesis of human zoonoses. J Infect Dis. 1994 Oct;170(4):918-26. doi: 10.1093/infdis/170.4.918. PMID: 7930737.

Hotez, PJ, D Diemert, KM Bacon, et al. 2013. The Human Hookworm Vaccine. Vaccine 31:B227-B232.

Howe, K. L., Bolt, B. J., Shafie, M., Kersey, P., \& Berriman, M. (2017). WormBase ParaSite- a comprehensive resource for helminth genomics. Molecular and biochemical parasitology, 215, 2-10.

Hrabar J, Trumbić Ž, Boc $\square$ ina I, Bus`elić I, Vrbatović A, Mladineo I (2019) Interplay between proinflammatory cytokines, miRNA, and tissue lesions in Anisakis-infected Sprague-Dawley rats. PLoS Negl Trop Dis 13(5): e0007397. https://doi. org/10.1371/journal.pntd.0007397 
Huber W, Carey VJ, Gentleman R, Anders S, Carlson M, Carvalho BS, et al. Orchestrating high-throughput genomic analysis with Bioconductor. Nat Methods (2015) 12:115-21. doi: 0.1038/nmeth.3252

Hunt VL, Hino A, Yoshida A, Kikuchi T. 2018. Comparative transcriptomics gives insights into the evolution of parasitism in Strongyloides nematodes at the genus, subclade and species level. Scientific RePoRTs | (2018) 8:5192 | DOI:10.1038/s41598-018-23514-Z

in silico analysis of the glutamate dehydrogenases of Teladorsagia circumcincta and International Helminth Genomes Consortium., Coghlan, A., Tyagi, R. et al. Comparative genomics of the major parasitic worms. Nat Genet 51, 163-174 (2019). https://doi.org/10.1038/s41588-018-0262-1

Iwona Polak 1, Elz $\square$ bieta Łopien' ska-Biernat 1,*, Robert Stryin'ski 1 , Jesús Mateos 2 and Mónica Carrera 2020. Comparative Proteomics Analysis of Anisakis simplex s.s.-Evaluation of the Response of Invasive Larvae to Ivermectin. Genes 2020, 11, 710; doi:10.3390/genes11060710

Janet Storm Jan Perner1, Isabela Aparicio2, Eva-Maria Patzewitz1,4, Kellen Olszewski3, Manuel Llinas3, Paul C Engel 2 and Sylke Müller Plasmodium falciparum glutamate dehydrogenase a is dispensable and not a drug target during erythrocytic development. Malaria Journal 2011 10:193. doi:10.1186/14752875-10-193.

Jiang P, Zao YJ, Yan SW, Song YY, Yang DM, Dai LY, Liu RD, Zhang X, Wang ZQ, Cui J. 2019. Molecular characterization of a Trichinella spiralis enolase and its interaction with the host's plasminogen. 50:106 https://doi.org/10.1186/s13567-019-0727-y

Joachim, J. et al. Centriolar Satellites Control GABARAP Ubiquitination and GABARAP-Mediated Autophagy. Curr Biol 27, 2123-2136 e2127, https://doi.org/10.1016/j.cub.2017.06.021 (2017)

Jones, P., Binns, D., Chang, H. Y., Fraser, M., Li, W., McAnulla, C., ... \& Hunter, S. (2014). InterProScan 5: genome-scale protein function classification. Bioinformatics, 30(9), 1236-1240.

Kaminsky, R., Ducray, P., Jung, M. et al. A new class of anthelmintics effective against drug-resistant nematodes. Nature 452, 176-180 (2008). https://doi.org/10.1038/nature06722

Katoh K, Standley DM. 2014.MAFFT: iterative refinement and additional methods. Methods Mol Biol. 1079:131-146.

Kearse, M., Moir, R., Wilson, A., Stones-Havas, S., Cheung, M., Sturrock, S., et al. (2012). Geneious basic: an integrated and extendable desktop software platform for the organization and analysis of sequence data. Bioinformatics 28, 1647-1649. doi: 10.1093/bioinformatics/bts199

Kim, J-H, J-O Kim, C-H Jeon, UH Nam, S Subramaniyam, S-I Yoo, J-H Park. 2018. Comparative transcriptome analyses of the third and fourth stage larvae of Anisakis simplex (Nematoda: Anisakidae). Molecular and Biochemical Parasitology 226:24-33.

Koehler, JW, ME Morales, BD Shelby, PJ Brindley. 2007. Aspartic protease activities of schistosomes cleave mammalian hemoglobins in a host-specific manner. Memórias do Instituto Oswaldo Cruz 102:83-85.

Kola, I, J Landis. 2004. Can the pharmaceutical industry reduce attrition rates? Nature Reviews Drug Discovery 3:711-716.

Kopylova E., Noé L. and Touzet H., "SortMeRNA: Fast and accurate filtering of ribosomal RNAs in metatranscriptomic data", Bioinformatics (2012), doi: 10.1093/bioinformatics/bts611.

Kotze, AC, AP Ruffell, AB Ingham. 2014. Phenobarbital induction and chemical synergism demonstrate the role of UDP-glucuronosyltransferases in detoxification of naphthalophos by Haemonchus contortus larvae. Antimicrob Agents Chemother 58:7475-7483.

Kumar S, Stecher G, Li M, Knyaz C, and Tamura K (2018) MEGA X: Molecular Evolutionary Genetics Analysis across computing platforms. Molecular Biology and Evolution 35:1547-1549.

Laing, R., Kikuchi, T., Martinelli, A. et al. The genome and transcriptome of Haemonchus contortus, a key model parasite for drug and vaccine discovery. Genome Biol 14, R88 (2013). https://doi.org/10.1186/gb-2013-14-8-r88

Langmead B, Wilks C, Antonescu V, Charles R. Scaling read aligners to hundreds of threads on generalpurpose processors. Bioinformatics. 2018 Jul 18. doi: 10.1093/bioinformatics/bty648. 
Laskowski, R.A., Rullmann, J.A.C., MacArthur, M.W., Kaptein, R., Thornton, J.M. (1996). AQUA and PROCHECK-NMR: programs for checking the quality of protein structures solved by NMR, J. Biomol. NMR 8, 477-486.

Lee J-D, Chung L-Y, Lin R-J, Wang J-J, Tu H-P, Yen C-M. Excretory/secretory proteases and mechanical movement of Anisakis pegreffii infective larvae in the penetration of BALB/c mice gastroin- testine. Kaohsiung J Med Sci. Published by Elsevier Taiwan LLC; 2017; 33: 594-601. https://doi.org/ 10.1016/j.kjms.2017.08.002.

Lee Robertson 1,2,*, Susana C. Arcos 1, Sergio Ciordia 3 , Noelia Carballeda-Sanguiao 4,5, María del Carmen Mena 3, Isabel Sánchez-Alonso 4 , Miguel Gonzalez-Muñoz 5 , Mercedes Careche 4 and Alfonso Navas. 2020. Immunoreactive Proteins in the Esophageal Gland Cells of Anisakis Simplex Sensu Stricto Detected by MALDI-TOF/TOF Analysis. Genes 2020, 11, 683; doi:10.3390/genes11060683.

Leles, D., Gardner, S.L., Reinhard, K. et al. Are Ascaris lumbricoides and Ascaris suum a single species?. Parasites Vectors 5, 42 (2012). https://doi.org/10.1186/1756-3305-5-42

Lenney, JF. 1990. Separation and characterization of two carnosine-splitting cytosolic dipeptidases from hog kidney (carnosinase and non-specific dipeptidase). Biol Chem Hoppe Seyler 371:433-440.

Leung, A.K.L.; Gerlich, D.; Miller, G.; Lyon, C.; Lam, Y.W.; Lleres, D.; Daigle, N.; Zomerdijk, J.; Ellenberg, J.; Lamond, A.I. Quantitative kinetic analysis of nucleolar breakdown and reassembly during mitosis in live human cells. J. Cell Biol. 2004, 166, 787-800.

Levsen, A., Svanevik, C. S., Cipriani, P., Mattiucci, S., Gay, M., Hastie, L. C., ... Pierce, G. J. (2018). A survey of zoonotic nematodes of commercial key fish species from major European fishing groundsIntroducing the FP7 PARASITE exposure assessment study. Fisheries Research, 202, 4-21. https://doi.org/10.1016/J.FISHRES.2017.09. 009.

Li W, Godzik A. Cd-hit: a fast program for clustering and comparing large sets of protein or nucleotide sequences. Bioinformatics. 2006;22:1658-9.

Li, B., Dewey, C.N. RSEM: accurate transcript quantification from RNA-Seq data with or without a reference genome. BMC Bioinformatics 12, 323 (2011). https://doi.org/10.1186/1471-2105-12-323

Llorens, C, SC Arcos, L Robertson, et al. 2018. Functional insights into the infective larval stage of Anisakis simplex s.s., Anisakis pegreffii and their hybrids based on gene expression patterns. BMC Genomics 19:592.

Love, M.I., Huber, W., Anders, S. (2014) Moderated estimation of fold change and dispersion for RNA-seq data with DESeq2. Genome Biology, 15:550. 10.1186/s13059-014-0550-8

Mairi C. Noverr, John R. Erb-Downward, Gary B. Huffnagle. Production of Eicosanoids and Other Oxylipins by Pathogenic Eukaryotic Microbes. Clinical Microbiology Reviews Jul 2003, 16 (3) 517 533; DOI: 10.1128/CMR.16.3.517-533.2003

Mannack L, Lane J 2015. The autophagosome: current understanding of formation and maturation. Research and Reports in Biochemistry 5: 39-58. http://dx.doi.org/10.2147/RRBC.S57405

Marino F, Lanteri G, Passantino A, De Stefano C, Costa A, Gaglio G, Macrì F. 2013. Experimental Susceptibility of Gilthead Sea Bream, Sparus aurata, via Challenge with Anisakis pegreffii Larvae. 2013, 701828, doi:10.1155/2013/701828

Marta Serrano-Moliner, María Morales-Suarez-Varela \& M. Adela Valero (2018): Epidemiology and management of foodborne nematodiasis in the European Union, systematic review 2000-2016, Pathogens and Global Health, DOI: 10.1080/20477724.2018.1487663

Matoušková, P, L Lecová, R Laing, et al. 2018. UDP-glycosyltransferase family in Haemonchus contortus: Phylogenetic analysis, constitutive expression, sex-differences and resistance-related differences. International Journal for Parasitology: Drugs and Drug Resistance 8:420-429.

Mattiucci, S, P Cipriani, A Levsen, M Paoletti, G Nascetti. 2018. Chapter Four - Molecular Epidemiology of Anisakis and Anisakiasis: An Ecological and Evolutionary Road Map. In: D Rollinson, JR Stothard, 
editors. Advances in Parasitology: Academic Press. p. 93-263.

https://doi.org/10.1016/bs.apar.2017.12.001.

McElwee, JJ, E Schuster, E Blanc, JH Thomas, D Gems. 2004. Shared Transcriptional Signature in Caenorhabditis elegans Dauer Larvae and Long-lived <em $>$ daf-2</em > Mutants Implicates Detoxification System in Longevity Assurance. Journal of Biological Chemistry 279:44533-44543.

Mi R, Yang X, Huang Y, Cheng L, Lu K, Han X, Chen Z 2017. Immunolocation and enzyme activity analysis of Cryptosporidium parvum enolase. Parasites \& Vectors (2017) 10:273 DOI 10.1186/s13071017-2200-y

Mladineo, I, Ž Trumbić, J Hrabar, A Vrbatović, I Bušelić, I Ujević, R Roje-Busatto, I Babić, C Messina. 2018. Efficiency of target larvicides is conditioned by ABC-mediated transport in the zoonotic nematode Anisakis pegreffii. Antimicrobial agents and chemotherapy 62.

Moneo I, Carballeda-Sangiao N, Gonza' lez-Muñoz M. New perspectives on the diagnosis of allergy to Anisakis spp. Curr Allergy Asthma Rep. 2017; 17: 27. https://doi.org/10.1007/s11882-017-0698-X PMID: 28429304

Moore, SA, AR Sielecki, MM Chernaia, NI Tarasova, MNG James. 1995. Crystal and Molecular Structures of Human Progastricsin at 1.62 ̊ Resolution. Journal of Molecular Biology 247:466-485.

Moriya, Y, M Itoh, S Okuda, AC Yoshizawa, M Kanehisa. 2007. KAAS: an automatic genome annotation and pathway reconstruction server. Nucleic Acids Res 35:W182-185. DOI: 10.1093/nar/gkm321.

Muñoz-Espín, D. and Serrano, M. (2014) Cellular senescence: from physiology to pathology. Nat. Rev. Mol. Cell Biol. 15, 482-496

Neelam Tia, Alok Kumar Singh 1, Poorti Pandey 1, Chandra Shekhar Azad 1, Pritee Chaudhary 1 , Indrajeet Singh Gambhir 2018. Role of Forkhead Box O (FOXO) transcription factor in aging and diseases Gene 30;648:97-105. doi: 10.1016/j.gene.2018.01.051

Nucleic Acids Res., 25 (1997), pp. 3389-3402

Nucleic Acids Research, Volume 47, Issue D1, 08 January 2019, Pages D506-D515, https://doi.org/10.1093/nar/gky1049

O Santos, L, A S Garcia-Gomes, M Catanho, C L Sodre, A LS Santos, M H Branquinha, C M d'Avila-Levy. 2013. Aspartic peptidases of human pathogenic trypanosomatids: perspectives and trends for chemotherapy. Current medicinal chemistry 20:3116-3133.

Ohsumi, Y. Historical landmarks of autophagy research. Cell research 24, 9-23, https://doi.org/10.1038/cr.2013.169 (2014).

Page, A.P. and I.L. Johnstone, The cuticle. WormBook, 2007: p. 1--15.

Palevich, N., Britton, C., Kamenetzky, L., Mitreva, M., de Moraes Mourão, M., Bennuru, S., Quack, T., Scholte, L.L.S., Tyagi, R., Slatko, B.E. (2018). Tackling Hypotheticals in Helminth Genomes. Trends in Parasitology. 34, 179-183. DOI: 10.1016/j.pt.2017.11.007.

Palevich, N., Maclean, P.H., Baten, A., Scott, R.W., Leathwick, D.M. (2019). The genome sequence of the anthelmintic-susceptible New Zealand Haemonchus contortus. Genome Biology and Evolution. 11(7), 1965-1970. DOI: 10.1093/gbe/evz141.

Palevich, N., Maclean, P.H., Choi, Y-J., Mitreva, M. (2020). Characterization of the complete mitochondrial genomes of two sibling species of parasitic roundworms, Haemonchus contortus and Teladorsagia circumcincta. Frontiers in Genetics. 11, 573395. DOI: 10.3389/fgene.2020.573395.

Parkinson J, Mitreva M, Whitton C, Thomson M, Daub J, Martin J, Schmid R, Hall N, Barrell B, Waterston RH, McCarter JP, Blaxter ML. 2004. A transcriptomic analysis of the phylum Nematoda. NATURE GENETICS 36, 1259-1267. doi:10.1038/ng1472

Philip Jones, David Binns, Hsin-Yu Chang, Matthew Fraser, Weizhong Li, Craig McAnulla, Hamish McWilliam, John Maslen, Alex Mitchell, Gift Nuka, Sebastien Pesseat, Antony F. Quinn, Amaia Sangrador-Vegas, Maxim Scheremetjew, Siew-Yit Yong, Rodrigo Lopez, and Sarah Hunter InterProScan 5: genome-scale protein function classification. Bioinformatics, Jan 2014 (doi:10.1093/bioinformatics/btu031) 
PMID: 20003500 PMCID: PMC2803857 DOI: 10.1186/1471-2105-10-421

PMID: 23104886 PMCID: PMC3530905 DOI: 10.1093/bioinformatics/bts635

Pozio E 2013. Integrating animal health surveillance and food safety: the example of Anisakis Rev. sci. tech. Off. int. Epiz., 2013, 32 (2), 487-496

Proteins, 57 (2004), pp. 702-710

Protocol for using Trinity for de novo transcriptome assembly and downstream analyses:

Qu Z, Li W, Zhang N, Li L, Yan H, Li T, Cui J, Yang Y, Jia W, Fu B. 2019. Comparative Genomic Analysis of Trichinella spiralis Reveals Potential Mechanisms of Adaptive Evolution Hindawi BioMed Research International Volume 2019, Article ID 2948973, 12 pages https://doi.org/10.1155/2019/2948973

Quiazon KMA, Yoshinaga Y, Ogawa K. 2011. Experimental challenge of Anisakis simplex sensu stricto and Anisakis pegreffii (Nematoda: Anisakidae) in rainbow trout and olive flounder. Parasitology International 60, 126-131 doi:10.1016/j.parint.2010.11.007

R Core Team (2020). R: A language and environment for statistical computing. R Foundation for Statistical Computing, Vienna, Austria. URL https://www.R-project.org/.

Rhoads ML, Fetterer RH, Urban JF. Release of hyaluronidase during in vitro development of Ascaris suum from the third to fourth larval stage. Parasitol Res (2001) 87:693-7. doi:10.1007/s00436010041

Robert Stryiński, Jesús Mateos, Santiago Pascual, Ángel F. González, José M. Gallardo,

Romero MC, Valero A, Navarro-Moll MC, Martın-Sanchez J. 2013. Experimental comparison of pathogenic potential of two sibling species Anisakis simplex s.s. and Anisakis pegreffii in Wistar rat. Tropical Medicine and International Health 18; 979-984.

S. Tripathy \& R. Sen \& S. K. Padhi \& S. Mohanty \& N. K. Maiti 2014. Upregulation of transcripts for metabolism in diverse environments is a shared response associated with survival and adaptation of Klebsiella pneumoniae in response to temperature extremes. Funct Integr Genomics (2014) 14:591-601 DOI 10.1007/s 10142-014-0382-3

S.F. Altschul, T.L. Madden, A.A. Schäffer, J. Zhang, Z. Zhang, W. Miller, D.J. LipmanGapped BLAST and PSI-BLAST: a new generation of protein database search programs

Scarff, Charlotte A., et al. "Structure of the protective nematode protease complex H-gal-GP and its conservation across roundworm parasites." PLoS pathogens 16.4 (2020): e1008465.

Schrodinger, 2010

Schrodinger, L. L. C. (2010). The PyMOL molecular graphics system. Version, 1(5), 0.

Sengupta, S.; Peterson, T.R.; Sabatini, D.M. Regulation of the mTOR complex 1 pathway by nutrients, growth factors, and stress. Mol. Cell 2010, 40, 310-322.

Serena Cavallero 1, Fabrizio Lombardo 1 , Marco Salvemini 2, Antonella Pizzarelli 1, Cinzia Cantacessi 3 and Stefano D'Amelio 2020. Comparative Transcriptomics Reveals Clues for Differences in Pathogenicity between Hysterothylacium aduncum, Anisakis simplex sensu stricto and Anisakis pegreffii Genes 2020, 11, 321; doi:10.3390/genes11030321

Shintre, Chitra A., et al. "Structures of ABCB10, a human ATP-binding cassette transporter in apo-and nucleotide-bound states." Proceedings of the National Academy of Sciences 110.24 (2013): 9710-9715. Simon Brown, Noorzaid Muhamad, Lisa R Walker, Kevin C Pedley, David C Simcock 2014. An Soneson C, Love MI, Robinson MD (2015). "Differential analyses for RNA-seq: transcript-level estimates improve gene-level inferences." F1000Research, 4. doi: 10.12688/f1000research.7563.1.

Sorci, G, B Faivre. 2009. Inflammation and oxidative stress in vertebrate host-parasite systems. Philos Trans R Soc Lond B Biol Sci 364:71-83.

Stamatakis A. 2014. RAxML version 8: a tool for phylogenetic analysis and post-analysis of large phylogenies. Bioinformatics 30(9):1312-1313.

STAR: Ultrafast Universal RNA-seq Aligner 
Stefano D'Amelio, Fabrizio Lombardo, Antonella Pizzarelli, Ilaria Bellini and Serena Cavallero 2020. Advances in Omic Studies Drive Discoveries in the Biology of Anisakid Nematodes Genes 2020, 11, 801; doi:10.3390/genes11070801

Stephen Altschul; Warren Gish; Webb Miller; Eugene Myers; David J. Lipman (1990). "Basic local alignment search tool". Journal of Molecular Biology. 215 (3): 403-410. doi:10.1016/S00222836(05)80360-2. PMID 2231712.

Stsiapanava, A., Tholander, F., Kumar, R. B., Qureshi, A. A., Niegowski, D., Hasan, M., ... \& RinaldoMatthis, A. (2014). Product formation controlled by substrate dynamics in leukotriene A4 hydrolase. Biochimica et Biophysica Acta (BBA)-Proteins and Proteomics, 1844(2), 439-446.

Susana C. Arcos, Sergio Ciordia, Lee Roberston, Inés Zapico, Yolanda Jiménez-Ruiz, Miguel GonzalezMuñoz, Ignacio Moneo, Noelia Carballeda-Sangiao, Ana Rodriguez-Mahillo, Juan P. Albar, and Alfonso Navas. 2014. Proteomic profiling and characterization of differential allergens in the nematodes Anisakis simplex sensu stricto and A. pegreffii. Proteomics Proteomics and Systems Biology 14, 15471568, doi:10.1002/pmic.201300529

T. Evans and N. Chapple: The animal health market. Nature Reviews Drug Discovery, 1(12), 937-938 (2002) doi:10.1038/nrd975

Tamimi, NA, P Ellis. 2009. Drug development: from concept to marketing! Nephron Clin Pract 113:c125131.

The Pfam protein families database in 2019: S. El-Gebali, J. Mistry, A. Bateman, S.R. Eddy, A. Luciani, S.C. Potter, M. Qureshi, L.J. Richardson, G.A. Salazar, A. Smart, E.L.L. Sonnhammer, L. Hirsh, L. Paladin, D. Piovesan, S.C.E. Tosatto, R.D. Finn. Nucleic Acids Research (2019) doi: 10.1093/nar/gky995

Unno, Hideaki, et al. "Structural basis for substrate recognition and hydrolysis by mouse carnosinase CN2." Journal of Biological Chemistry 283.40 (2008): 27289-27299.

Valentina Grossi, Candida Fasano, Valentina Celestini, Martina Lepore Signorile, Paola Sanese, Cristiano Simone 2019. Chasing the FOXO3: Insights into Its New Mitochondrial Lair in Colorectal Cancer Landscape Cancers 2019, 11, 414; doi:10.3390/cancers 11030414

Valeria Marzano 1, Stefania Pane 2 , Gianluca Foglietta 2, Stefano Levi Mortera 1, Pamela Vernocchi 1, Andrea Onetti Muda 3 and Lorenza Putignani 2020. Mass Spectrometry Based-Proteomic Analysis of Anisakis spp.: A Preliminary Study towards a New Diagnostic Tool Genes 2020, 11, 693; doi:10.3390/genes11060693

Van Thiel, P. H. (1960). Anisakis. Parasitology, 53(16), 4.

Viney M. 2018. The genomic basis of nematode parasitism. Briefings in Functional Genomics, 17(1), 8-14. doi: $10.1093 / \mathrm{bfgp} / \mathrm{elx} 010$

Wang T. 2014. Transcriptomic and proteomic analysis of Ascaris suum larvae during their hepato-tracheal migration. PhD thesis, Ghent University, pp. 143.

Waterhouse R.M., Seppey M., Simão F.A., Manni M., Ioannidis P., Klioutchnikov G., Kriventseva E.V., Zdobnov E.M. BUSCO applications from quality assessments to gene prediction and phylogenomics. Mol. Biol. Evol. 2018;35:543-548. doi: 10.1093/molbev/msx319.

Watts, JL, M Ristow. 2017. Lipid and Carbohydrate Metabolism in Caenorhabditis elegans. Genetics 207:413-446.

Wiederstein, M., Sippl, M.J. (2007). ProSA-web: interactive web service for the recognition of errors in three-dimensional structures of proteins, Nucleic Acids Res. 35, W407-W410.

Winter AD, McCormack G, Myllyharju J, Page AP. Prolyl 4-hydroxlase activity is essential for development and cuticle formation in the human infective parasitic nematode Brugia malayi. J Biol Chem (2013) 288:1750-61. doi:10.1074/jbc.M112.397604

Winter AD, McCormack G, Page AP. Protein disulfide isomerase activity is essential for viability and extracellular matrix formation in the nematode Caenorhabditis elegans. Dev Biol (2007) 308:449-61. doi:10.1016/j.ydbio.2007.05.041 
Y. Zhang, J. Skolnick Scoring function for automated assessment of protein structure template quality

Yang, J., Yan, R., Roy, A., Xu, D., Poisson, J., Zhang, Y. (2015). The I-TASSER Suite: Protein structure and function prediction. Nature Methods, 12: 7-8.

Young, M.D., Wakefield, M.J., Smyth, G.K., Oshlack, A., Gene ontology analysis for RNA-seq: accounting for selection bias, Genome Biology, 11, 2, Feb 2010, R14

Zaiss MM, Harris NL. Interactions between the intestinal microbiome and helminth parasites. Parasite Immunol. 2016; 38:5-11.

Zhang, Lin, et al. "Insight into tartrate inhibition patterns in vitro and in vivo based on cocrystal structure with UDP-glucuronosyltransferase 2B15." Biochemical pharmacology 172 (2020): 113753.

Zhang, S. 2019. Comparative Transcriptomic Analysis of the Larval and Adult Stages of Taenia pisiformis. Genes 10:507.

Zhang, Z, L Miao, X Xin, J Zhang, S Yang, M Miao, X Kong, B Jiao. 2014. Underexpressed CNDP2 Participates in Gastric Cancer Growth Inhibition through Activating the MAPK Signaling Pathway. Molecular Medicine 20:17-28.

Zhao, C, W Haase, R Tampé, R Abele. 2008. Peptide specificity and lipid activation of the lysosomal transport complex ABCB9 (TAPL). Journal of Biological Chemistry 283:17083-17091. A rat model of intragastric infection with Anisakis spp. live larvae: histopathological study. Parasi- tol Res. 2013; 112: 2409-2411. https://doi.org/10.1007/s00436-013-3359-6.

Zumerle S, Alimonti A. 2020. in and out from senescence. Nature Cell Biology. 22, 753-754 doi.org/10.1038/s41556-020-0540-x. 


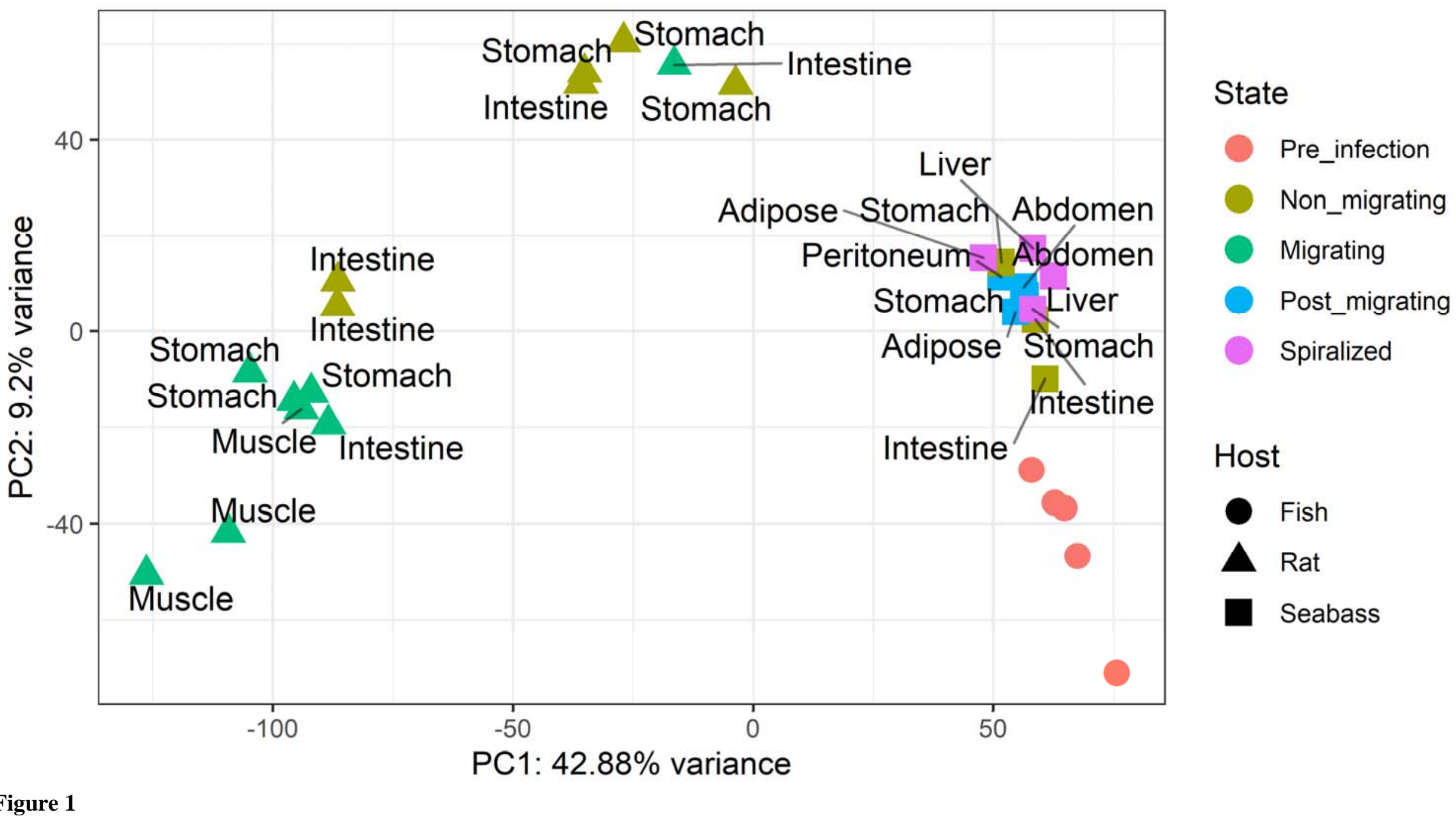

6 Figure 1 
Migrating

DEGs in Rat
Migrating

\section{DEGs in Seabass}

65

1539

10

323
297

112

64

Interaction 
k001110 Biosynthesis of secondary metabolites

ko03010 Ribosome ko01120 Microbial metabolism in diverse environments

ko00190 Oxidative phosphorylation

ko04723 Retrograde endocannabinoid signaling ko01230 Biosynthesis of amino acids

ko01200 Carbon metabolism

ko04142 Lysosome

ko01212 Fatty acid metabolism ko00480 Glutathione metabolism

ko04140 Autophagy - anima

ko00010 Glycolysis / Gluconeogenesis

ko03320 PPAR signaling pathwa

ko00982 Drug metabolism - cytochrome P

ko00980 Metabolism of xenobiotics by cytochrome $P$
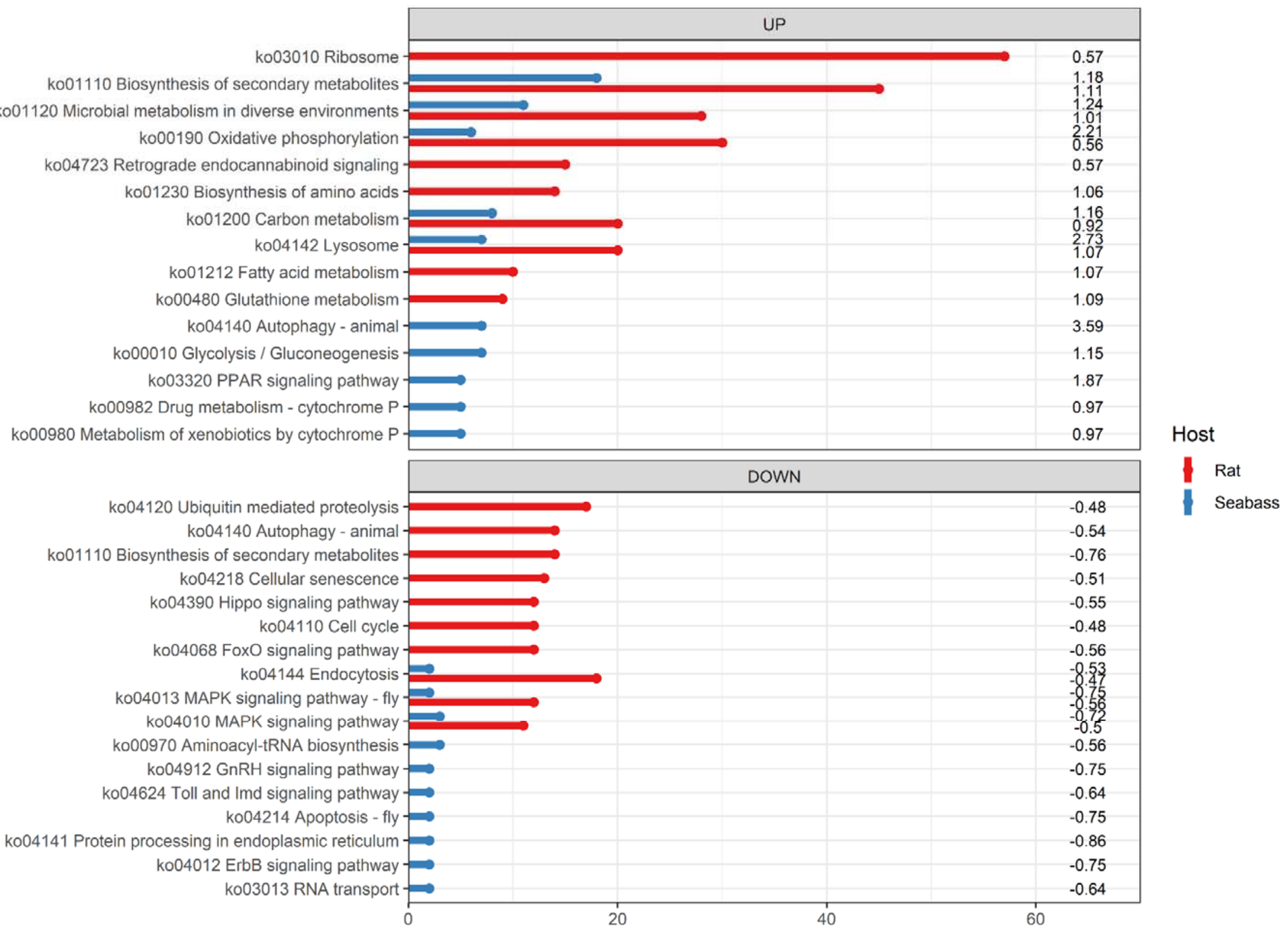


\section{ABCB9 UGT3 ASP6 LKHA4 CNDP2}

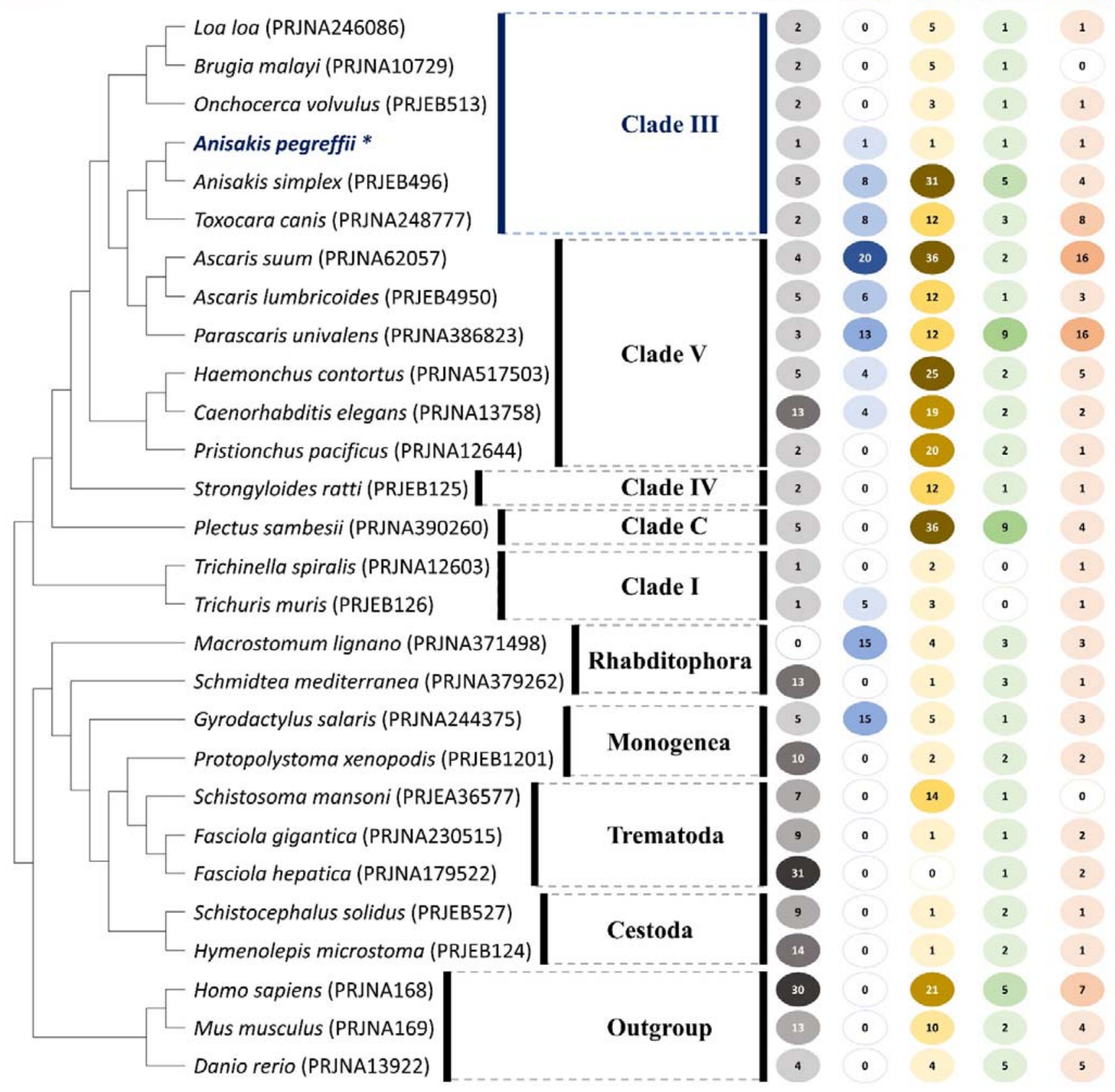

Figure 4 
bioRxiv preprint doi: https://doi.org/10.1101/2021.03.12.435207; this version posted March 13,2021. The copyright holder for this preprint (which was not certified by peer review) is the author/funder, who has granted bioRxiv a license to display the preprint in perpetuity. It is made available under aCC-BY-NC-ND 4.0 International license.
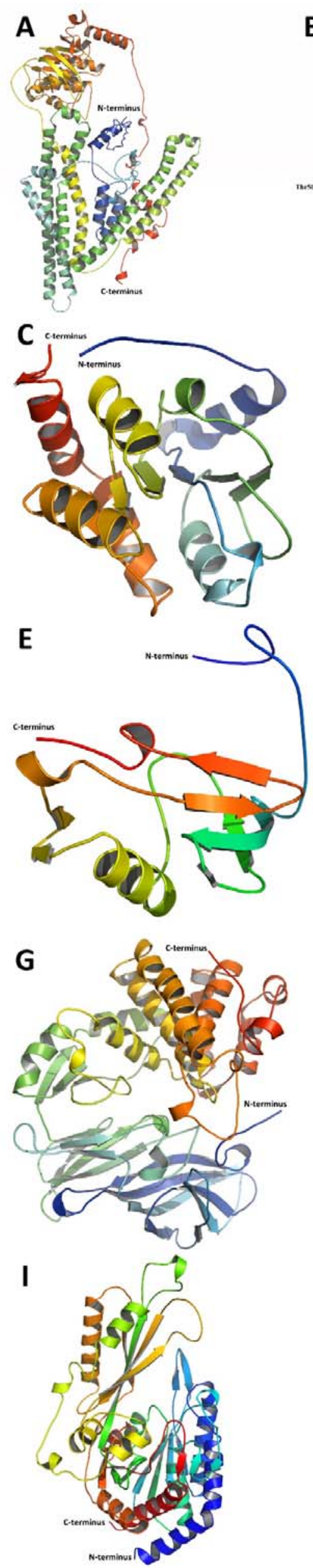
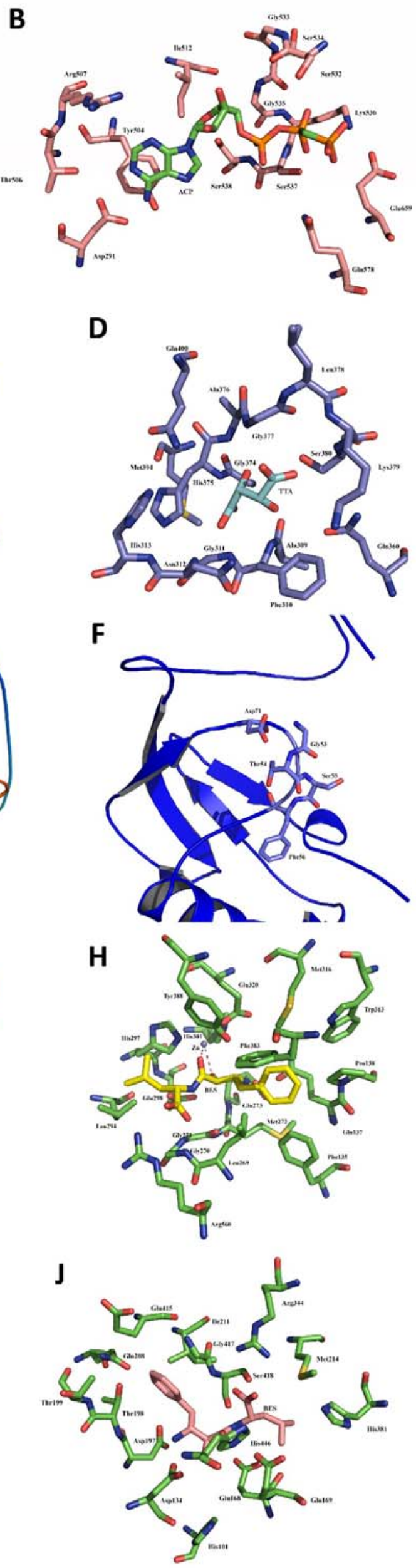


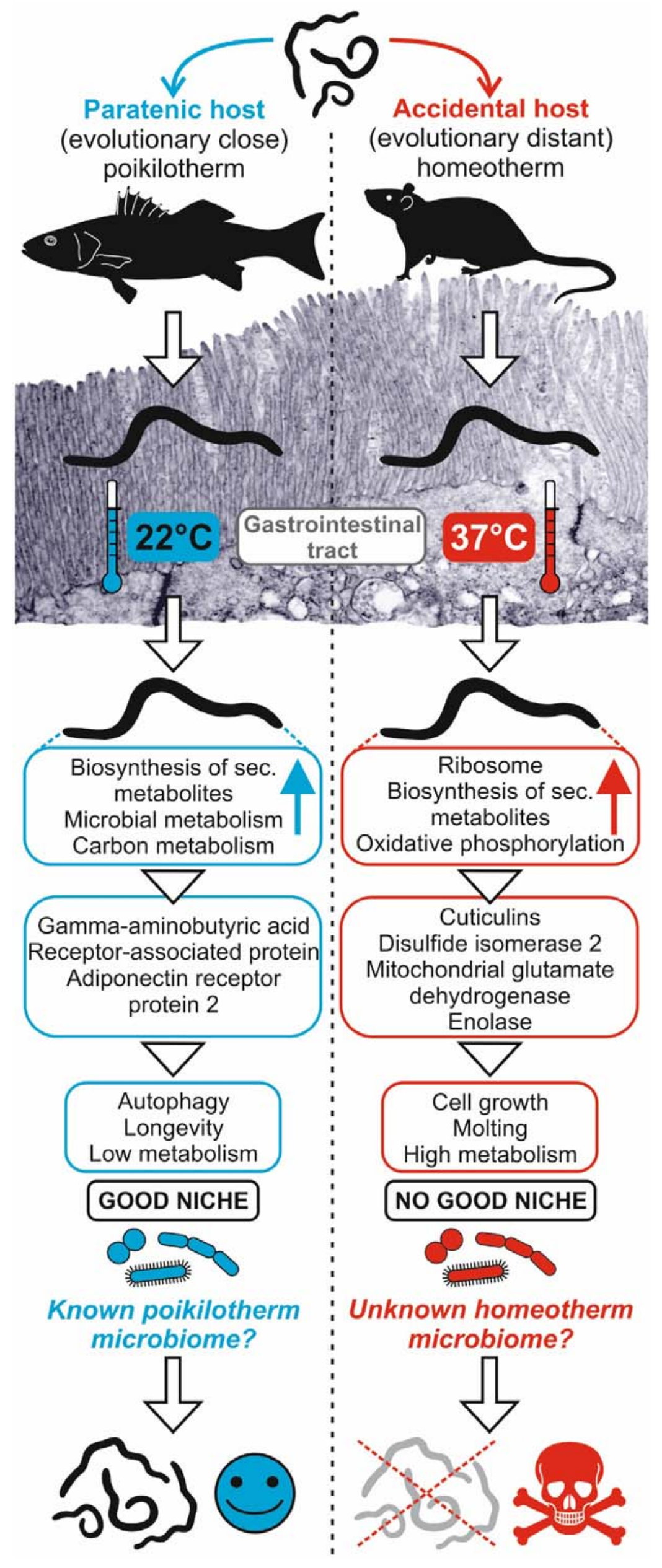

